\title{
A relaxation method via the Born-Infeld system
}

\author{
Michaël Baudin*†, Frédéric CoQUEL ${ }^{\ddagger}$, and Quang Huy Tran*§
}

December 22, 2007

\begin{abstract}
We propose an original relaxation scheme for scalar conservation laws of the form $\partial_{t} u+\partial_{x}(u(1-u) g(u))=0$, where $g \in \mathscr{C}^{1}([0,1] ; \mathbb{R})$. Those scalar conservation laws come from a drift-flux model for two-phase flows [16]. Unlike Jin and Xin's approach [12], the new relaxation strategy does not involve any tuning parameter, but makes use of the Born-Infeld system [5,18]. The advantage of this new method is that it enables us to achieve a maximum principle on the velocities $w=(1-u) g$ and $z=-u g$, and to control the sign of the numerical flux. Stability is established for a wide class of eligible functions $g$. This method can be plugged into a scheme for two-phase fluids, for which numerical experiments are shown.
\end{abstract}

\section{Keywords}

Two-phase flow, drift-flux model, multi-component fluid, relaxation methods, Born-Infeld system, Chapman-Enskog analysis, Whitham condition

\section{Introduction}

As a sequel to the previous works $[2,3]$ on relaxation methods for the simulation of twophase flows in pipelines, this paper is a first step toward the multi-component case. More specifically, it shows how a numerical difficulty that arises in a large two-phase multicomponent system can be most adequately solved by some unexpected theoretical developments for a scalar conservation law.

For the sake of simplicity, let us use Lagrangian mass coordinates to introduce the problem. In $[2,3]$, we proposed to solve the drift-flux model $[16,17]$

$$
\begin{aligned}
& \partial_{t} \tau-\partial_{m} v=0 \\
& \partial_{t} v+\partial_{m} P=0 \\
& \partial_{t} Y-\partial_{m} \sigma=0,
\end{aligned}
$$

*Département Mathématiques Appliquées, Institut Français du Pétrole, 1 et 4 avenue de Bois-Préau, 92852 Rueil-Malmaison Cedex, France

† presently at: ALTRAN Aeronautics-Space \& Defence, 2 rue Paul Vaillant Couturier, 92300 LevalloisPerret, France

${ }^{\ddagger}$ Laboratoire Jacques-Louis Lions, CNRS et Université Pierre et Marie Curie, B.C. 187, 75252 Paris Cedex 05, France

${ }^{\S}$ Corresponding author: Q-Huy.Tran@ifp.fr, phone +3314752 74 22, fax +3314752 7022 
by the relaxation model

$$
\begin{aligned}
\partial_{t} \tau-\partial_{m} v & =0 \\
\partial_{t} v+\partial_{m} \Pi & =0 \\
\partial_{t} \Pi+a^{2} \partial_{m} v & =\lambda(P-\Pi) \\
\partial_{t} Y-\partial_{m} \Sigma & =0 \\
\partial_{t} \Sigma-b^{2} \partial_{m} Y & =\lambda(\sigma-\Sigma) .
\end{aligned}
$$

In (1.1), the slip

$$
\sigma=\rho Y(1-Y) \phi(\tau, Y, v)
$$

and the total pressure

$$
P=p(\tau, Y)+\rho Y(1-Y) \phi^{2}(\tau, Y, v)
$$

are functions of the specific volume $\tau=\rho^{-1} \in \mathbb{R}_{+}^{*}$, the gas mass-fraction $Y \in[0,1]$ and the mixture velocity $v$. The thermodynamic pressure $p$ in (1.4) and the hydrodynamic slip velocity $\phi$ in (1.3) are algebraic closure laws.

The relaxation method consists in replacing the highly nonlinear quantities $P$ and $\sigma$ by the new variables $\Pi$ and $\Sigma$, to which (1.2c) and (1.2e) are imposed as governing equations Note that the subsystem (1.2d)-(1.2e) can be seen as the Jin-Xin relaxation [12] applied to $(1.1 \mathrm{c})$, considered as a "scalar" conservation law on $Y$. The tuning parameters $a, b$ are at our disposal in order to stabilize the method. It was shown in $[2,3]$ that under the Whitham conditions

$$
\begin{aligned}
a^{2} & >-P_{\tau}+P_{v}^{2} \\
b & >\left|\sigma_{Y}\right|,
\end{aligned}
$$

the relaxation system (1.2) is a "good" approximation to the original system (1.1) in the limit $\lambda \rightarrow \infty$. Furthermore, at the discrete level, when the variables are updated by a first-order explicit scheme, we are in a position to guarantee a positivity principle for the specific volume and the mass-fraction, namely,

$$
\tau>0 \text { and } Y \in[0,1]
$$

as soon as $a, b$ are larger than some known and computable bounds. This is the main advantage of relaxation over other numerical schemes $[7,8,15]$.

To take into account the multi-component nature of the flow, we want to add two additional balance laws to model (1.1), namely,

$$
\begin{aligned}
\partial_{t}(Y \xi)-\partial_{m}(\xi \sigma) & =0 \\
\partial_{t}((1-Y) \eta)+\partial_{m}(\eta \sigma) & =0 .
\end{aligned}
$$

The variables $(\xi, \eta) \in[0,1]^{2}$ are the partial fractions of some component in the gas and liquid phase, and do appear as new arguments of $P$ and $\sigma$. Combining (1.7) with (1.1c), we get

$$
\begin{aligned}
& \partial_{t} \xi-\frac{\sigma}{Y} \partial_{m} \xi=0 \\
& \partial_{t} \eta+\frac{\sigma}{1-Y} \partial_{m} \eta=0
\end{aligned}
$$


which shows that $-\sigma / Y$ and $\sigma /(1-Y)$ are characteristic speeds of the system (1.1), (1.8). The relaxation version of (1.7) is

$$
\begin{aligned}
\partial_{t}(Y \xi)-\partial_{m}(\xi \Sigma) & =0 \\
\partial_{t}((1-Y) \eta)+\partial_{m}(\eta \Sigma) & =0 .
\end{aligned}
$$

Combining (1.9) with (1.2d), we get

$$
\begin{aligned}
& \partial_{t} \xi-\frac{\Sigma}{Y} \partial_{m} \xi=0 \\
& \partial_{t} \eta+\frac{\Sigma}{1-Y} \partial_{m} \eta=0,
\end{aligned}
$$

which shows that $-\Sigma / Y$ and $\Sigma /(1-Y)$ are characteristic speeds of the system (1.2), (1.10). The trouble with this relaxation strategy is that when we need to know the velocities

$$
-\frac{\Sigma^{*}}{Y^{*}} \text { and } \frac{\Sigma^{*}}{1-Y^{*}}
$$

of the intermediate state (in the solution of the Riemann problem associated with the $(Y, \Sigma)$-subsystem), we are faced with a division problem between two vanishing numbers. The handling of this problem proves to be awkward, especially in the neighborhood of single-phase states where $Y^{*} \rightarrow 0$ or 1 .

It could be argued that the actual update of $\xi, \eta$ by (1.9) does not require the velocities (1.11). The only thing we need to know is $\Sigma^{*}$, the sign of which is sufficient to decide about the upwind values for $\xi, \eta$. While this argument is true for an explicit scheme, in which case the relaxation (1.9) coincides with Larrouturou's decoupling device [13], it falls flat if our ultimate goal to work out an implicit scheme in the fashion of Baudin et al. [3]. Indeed, in order to interpret the scheme as a Roe method, we have to be able to compute the speeds (1.11) in an efficient way. A final, albeit accidental observation is that the $\Sigma^{*}$ coming from Jin-Xin's method (1.2d)-(1.2e) for (1.1c) may have the "wrong" sign when $b$ is too large (see $\S 3$ for details).

In the present work, we would like to put forward a new relaxation approach to the "scalar" equation (1.1c), the interest of which is to ensure a maximum principle on the values of the Lagrangian velocities $\rho(1-Y) \phi$ and $-\rho Y \phi$ for the intermediate state, and to secure the correct sign for the final numerical flux $\Sigma^{*}$. To the astonishment of ourselves, the new relaxation philosophy resorts to a system of equations studied by Born and Infeld [5] about a century ago in electrodynamics! This is why we also call it the Born-Infeld relaxation for short.

This paper is organized as follows. First, in $\S 2$, the Born-Infeld relaxation is presented in its purest essence for the scalar case, along with its numerous and elegant properties. This is most fundamental part of our contribution, the theoretical results of which are carried over to an arbitrary flux function in Appendix A. Then, in $\S 3$, we show how to integrate this scalar method into a more intricate relaxation scheme for the full drift-flux model in Lagrangian coordinates. The case of Eulerian coordinates is addressed in $\S 4$, where numerical results are supplied in order to compare the new relaxation scheme to the standard one.

\section{Relaxation via Born-Infeld for scalar conservation laws}

Let us start by assuming that $\rho=1$ and $\phi=\phi(Y)$, which allows us to consider (1.1c) as scalar conservation law. In order to comply with the usual notations for hyperbolic 
equations and to highlight the abstract nature of the Born-Infeld relaxation method, we proceed to the change of notations

$$
Y \rightarrow u, \quad-\sigma \rightarrow f, \quad-\phi \rightarrow g, \quad m \rightarrow x
$$

We consider the conservation law (1.1c), rewritten as

$$
\partial_{t} u+\partial_{x} f(u)=\partial_{t} u+\partial_{x}(u(1-u) g(u))=0
$$

and defined for $u \in[0,1]$ under the hypothesis $g \in \mathscr{C}^{1}([0,1] ; \mathbb{R})$. In particular, $g$ is bounded. Equation (2.2) can be thought of as two nonlinear convective equations

$$
\partial_{t}(u)+\partial_{x}(u \cdot w(u))=0 \quad \text { or } \quad \partial_{t}(1-u)+\partial_{x}((1-u) \cdot z(u))=0
$$

with the apparent velocities

$$
w(u)=(1-u) g(u)=\frac{f(u)}{u} \quad \text { and } \quad z(u)=-u g(u)=-\frac{f(u)}{1-u} .
$$

The difference

$$
g(u)=w(u)-z(u)
$$

then appears to be the slip velocity between the phase $u$ and the phase $1-u$. By inverting (2.4), we have

$$
u=\frac{z(u)}{z(u)-w(u)} \quad \text { and } \quad f(u)=\frac{w(u) z(u)}{z(u)-w(u)}
$$

provided that $w(u) \neq z(u)$.

\subsection{Change of variables}

As we seek to ensure a monotonicity principle for the phase velocities, it is natural to choose them as evolution variables. Let us introduce the pair of independent variables $(W, Z)$, meant to be the relaxation counterparts of the equilibrium values $(w(u), z(u))$. The formulae (2.6) suggest to define the pair $(U, F)$, the relaxation counterparts of the equilibrium values $(u, f(u))$, as

$$
U(W, Z)=\frac{Z}{Z-W} \quad \text { and } \quad F(W, Z)=\frac{W Z}{Z-W}
$$

for $W \neq Z$. In the same spirit as $(2.1)$, the variable $F$ is the new notation for $-\Sigma$. The inverse transformation of $(2.7)$ is

$$
W(U, F)=\frac{F}{U} \quad \text { and } \quad Z(U, F)=-\frac{F}{1-U} .
$$

In imitation of (2.5), we also define

$$
G(W, Z)=W-Z
$$

to be the relaxation version of the slip velocity $g(u)$. Then,

$$
F=U(1-U) G, \quad W=(1-U) G, \quad Z=-U G .
$$


Our first task is to clarify the ranges of the variables involved so that the transformations (2.7)-(2.8) make sense and so that we can make the identification $U \equiv u \in[0,1]$. Let $\Omega \subset \mathbb{R}^{2}$ be the $(W, Z)$-domain defined as

$$
\Omega=\left\{\mathbb{R}_{-} \times \mathbb{R}_{+}\right\} \cup\left\{\mathbb{R}_{+} \times \mathbb{R}_{-}\right\} \backslash(0,0)
$$

and let $\mho \subset[0,1] \times \mathbb{R}$ be the $(U, F)$-domain defined as

$$
\mho=] 0,1[\times \mathbb{R} \cup(0,0) \cup(1,0) .
$$

Proposition 2.1. The pair $(U(W, Z), F(W, Z))$ introduced in (2.7) is well-defined for all $(W, Z) \in \Omega$. The domain of $F$ can be extended to $\bar{\Omega}=\Omega \cup(0,0)$ by continuity by setting

$$
F(0,0)=0
$$

The pair $(W(U, F), Z(U, F))$ introduced in (2.8) is well-defined for $(U, F) \in \mho^{\circ}=\mho \backslash$ $\{(0,0) \cup(1,0)\}$. The domain of $(W, Z)$ can be extended to $\mho$ by continuity along the equilibrium manifold $F=f(U)$ by setting

$$
(W(0,0), Z(0,0))=(w(0), z(0)) \quad \text { and } \quad(W(1,0), Z(1,0))=(w(1), z(1)) .
$$

Proof From the first part of (2.7), we have

$$
U(1-U)=-\frac{W Z}{(Z-W)^{2}} .
$$

Hence, $U \in[0,1]$ if and only if $W Z \leq 0$, which means that $(W, Z) \in \bar{\Omega}$. Then, the pair $(U, F)$ is well-defined by $(2.7)$ if and only if $W \neq Z$, which amounts to $(W, Z) \in \Omega$. At the origin, $F$ can be extended by (2.13) because

$$
\lim _{(W, Z) \in \Omega \rightarrow(0,0)} F(W, Z)=0 .
$$

It is not possible to do the same for $U$, but this does not cause any harm to the method.

If $(U, F) \in \mho^{\circ}$, there is no problem in computing $(W, F)$ via $(2.8)$. We cannot do this for $U=0$ or 1 , but if $F=f(U)=U(1-U) g(U)$, then simplifications occur, which enables us to define $(W, Z)$ at $(U, F)=(0,0)$ and $(1,0)$.

The key fact on which we wish to draw attention is that $F$ can be defined continuously over $\bar{\Omega}$, so that the Born-Infeld flux is always well-defined (see Theorem 2.5) and therefore the new relaxation method can be applied even for single-phase states $U=0$ or 1 .

\subsection{Relaxation system}

Definition 2.1. The Born-Infeld relaxation system for the scalar conservation law (2.2) is defined as

$$
\begin{aligned}
& \partial_{t} W+Z \partial_{x} W=\lambda[w(U(W, Z))-W] \\
& \partial_{t} Z+W \partial_{x} Z=\lambda[z(U(W, Z))-Z],
\end{aligned}
$$

for $(W, Z) \in \Omega$, where $\lambda>0$ is the relaxation coefficient. 
This name is justified by the fact that when $\lambda=0$, system (2.17) coincides with Serre's form [18] of the Born-Infeld equations. The historical Born-Infeld system [5,19] stems from quantum mechanics and is actually more sophisticated. To our knowledge, this is the first time that it appears in the design of relaxation schemes, a context totally unrelated to field theory.

It seems to be the first time too that a nonlinear combination of the relaxation equations must be used in order to recover the "original" equation, as indicated in the following Lemma.

Lemma 2.1. For all $\lambda>0$, smooth solutions of (2.17) are also solution of

$$
\partial_{t} U(W, Z)+\partial_{x} F(W, Z)=0 .
$$

Proof Since smooth solutions are differentiable, we multiply $(2.17 \mathrm{a})$ by $U_{W}=Z G^{-2}$, (2.17b) by $U_{Z}=-W G^{-2}$ and add them together. It is readily checked that the left-hand side of the sum is equal to $\partial_{t} U+\partial_{x} F$. As for the right-hand side, it also vanishes thanks to

$$
Z(w(U)-W)-W(z(U)-Z)=Z w(U)-W z(U)=g(U)[(1-U) Z+U W]
$$

and because of (2.7).

For the moment, the property (2.18) holds true only for smooth solutions. In order to extend it to weak solutions, we simply need to have a linear degeneracy of the characteristic fields of (2.17).

Proposition 2.2. The eigenvalues $(Z, W)$ of (2.17), both linearly degenerate, are respectively associated with the strong Riemann invariants $W$ and $Z$.

Proof See [18].

This expected degeneracy is in accordance with the orthodoxy in the area of relaxation systems $[6,10,11]$. The fact that all characteristic fields are degenerate by construction enables us to state a more complete equivalent result.

Theorem 2.1. For all $\lambda>0$, the Born-Infeld relaxation system (2.17) is equivalent to the system

$$
\begin{aligned}
\partial_{t} U+\quad \partial_{x}(U(1-U) G) & =0 \\
\partial_{t} G+G^{2} \partial_{x} U & =\lambda[g(U)-G] .
\end{aligned}
$$

in the sense of weak solutions, where $(U, G) \in[0,1] \times \mathbb{R}$ is considered as a pair of independent variables.

Proof Thanks to linear degeneracy, we can restrict ourselves to smooth solutions. Equation (2.20a) is none other than (2.18) of Lemma 2.1. As for (2.20b), it can obtained by subtracting (2.17b) to (2.17a) and by arguing that

$$
Z \partial_{x} W-W \partial_{x} Z=\left(Z W_{U \mid G}-W Z_{U \mid G}\right) \partial_{x} U+\left(Z W_{G \mid U}-W Z_{G \mid U}\right) \partial_{x} G
$$

in the left-hand side.

In preparation for the generalization of the Born-Infeld to an arbitrary flux function (see Appendix), we point out the following property. 
Corollary 2.1. The Born-Infeld relaxation system satisfies the additional equation

$$
\partial_{t}((1-2 U) G)-\partial_{x}\left(U(1-U) G^{2}\right)=\lambda(1-2 U)[g(U)-G]
$$

the left-hand side of which is in conservation form.

Proof Let $I=(1-2 U) G$ and $K=-U(1-U) G^{2}$. In terms of $(W, Z)$, we have $I=W+Z$ and $K=W Z$. Adding (2.17a) and (2.17b) together, we obtain the desired equality

$$
\partial_{t} I+Z \partial_{x} W+W \partial_{x} Z=\lambda\{(1-u)-u\}[g(U)-G] .
$$

because $Z \partial_{x} W+W \partial_{x} Z=\partial_{x}(W Z)=\partial_{x} K$.

\subsection{Chapman-Enskog analysis and Whitham condition}

We now show the extent to which the Born-Infeld relaxation system (2.17), or its equivalent form (2.20), is actually a "good" approximation to the original equation (2.2).

Theorem 2.2. At the first order approximation in $\lambda^{-1}$, the solution $u$ to relaxation system (2.17) or 2.20) satisfies the equivalent equation

$$
\partial_{t} u+\partial_{x} f(u)=\lambda^{-1} \partial_{x}\left\{-\left[f^{\prime}(u)-w(u)\right]\left[f^{\prime}(u)-z(u)\right] \partial_{x} u\right\} .
$$

Proof With the identification $u \equiv U$, we insert the standard Chapman-Enskog expansion

$$
G=g(u)+\lambda^{-1} g_{1}+O\left(\lambda^{-2}\right)
$$

into (2.20b). Keeping the leading terms only, we obtain

$$
-g_{1}=\partial_{t} g(u)+g^{2}(u) \partial_{x} u .
$$

Arguing that $\partial_{t} u=-\partial_{x} f(u)$ is valid at the zeroth-order, we transform the right-hand side into

$$
-g_{1}=\left[g^{2}(u)-g^{\prime}(u) f^{\prime}(u)\right] \partial_{x} u,
$$

Now, plugging (2.25) into the first equation of (2.20), moving first-order terms to the right-hand side and using (2.27), we end up with

$$
\begin{aligned}
\partial_{t} u+\partial_{x} f(u) & =\lambda^{-1} \partial_{x}\left\{-u(1-u) g_{1}\right\} \\
& =\lambda^{-1} \partial_{x}\left\{u(1-u)\left[g^{2}(u)-g^{\prime}(u) f^{\prime}(u)\right] \partial_{x} u\right\} .
\end{aligned}
$$

Let us rearrange the kernel

$$
D(u)=u(1-u)\left[g^{2}(u)-g^{\prime}(u) f^{\prime}(u)\right] .
$$

On one hand,

$$
u(1-u) g^{2}(u)=-w(u) z(u) .
$$

On the other hand, multiplying the equality $f^{\prime}=(1-2 u) g+u(1-u) g^{\prime}$ by $f^{\prime}$ and isolating the product $g^{\prime} f^{\prime}$, we have

$$
\begin{aligned}
u(1-u) g^{\prime}(u) f^{\prime}(u) & =\left[f^{\prime}(u)\right]^{2}-(1-2 u) g(u) f^{\prime}(u) \\
& =\left[f^{\prime}(u)\right]^{2}-(w(u)+z(u)) f^{\prime}(u) .
\end{aligned}
$$

Inserting (2.30) and (2.31) into (2.29) yields

$$
\begin{aligned}
D(u) & =-w(u) z(u)+(w(u)+z(u)) f^{\prime}(u)-\left[f^{\prime}(u)\right]^{2} \\
& =-\left[f^{\prime}(u)-w(u)\right]\left[f^{\prime}(u)-z(u)\right],
\end{aligned}
$$

which completes the proof. 
Theorem 2.3. A sufficient condition for the relaxation system (2.20) to be a dissipative approximation of the original equation (2.2) is that the Whitham subcharacteristic condition

$$
f^{\prime}(u) \in\lfloor w(u), z(u)\rceil,
$$

holds for all $u$ in the range of the problem at hand.

Proof At the continuous level, the dissipative approximation means that the kernel $D(u)$ in the equivalent equation $(2.24)$ be a diffusive kernel, i.e., $D(u) \geq 0$. This amounts to

$$
\left[f^{\prime}(u)-w(u)\right]\left[f^{\prime}(u)-z(u)\right] \leq 0,
$$

hence (2.33).

Rigorously speaking, we should have required $D(u)>0$ at the PDE level. At the discrete level, however, we will see in $\S 2.5$ that $D(u) \geq 0$ is sufficient to guarantee the monotonicity of the numerical flux.

Unsurprisingly, the Whitham condition demands that the eigenvalue of the original equation be enclosed by the two eigenvalues of the relaxation system [19]. The novel feature is that, in the present method, condition (2.33) may appear to be quite severe: only a class of flux functions $f$ are eligible for the Born-Infeld relaxation. Let us investigate further into this class of flux functions. The boundedness of $g$ implies $f(0)=f(1)=0$, and we can write

$$
w(u)=\frac{f(u)-f(0)}{u-0} \quad \text { and } \quad z(u)=\frac{f(1)-f(u)}{1-u} .
$$

From (2.35), we infer the geometrical interpretation depicted in Fig. 1. On the curve $\mathscr{C}$ given by the Cartesian equation $f=f(u)$, let $\mathrm{M}=(u, f)$ be a running point, $\mathrm{A}=(0,0)$ and $\mathrm{B}=(1,0)$. Then, $w$ is the slope of $\mathrm{AM}$, while $z$ is that of BM. Condition $(2.33)$ amounts to saying that the tangent to $\mathscr{C}$ at $\mathrm{M}$ lies outside the angular sector $\angle(\mathrm{MA}, \mathrm{MB})$.

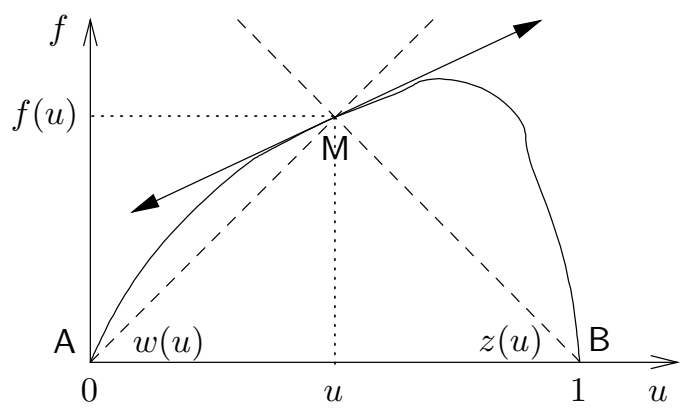

Figure 1: Geometrical interpretation of the Whitham condition.

For $u=0$ or 1 , the Whitham condition holds with equality, since $f^{\prime}(0)=w(0)$ and $f^{\prime}(1)=z(1)$. We are going to characterize the Whitham condition for a local point $u \in] 0,1[$ in two different ways. The first way is a condition in terms of $w$ and $z$ alone.

Lemma 2.2. Let $u \in] 0,1[$. The Whitham condition (2.33) is satisfied at $u$ if and only if the functions $w$ and $z$ are decreasing at $u$ when $f(u)>0$, increasing at $u$ when $f(u)<0$, and critical when $f(u)=0$. 
Proof A straightforward computation shows that

$$
w^{\prime}=\frac{u f^{\prime}-f}{u^{2}} \quad \text { and } \quad z^{\prime}=-\frac{(1-u) f^{\prime}+f}{(1-u)^{2}} .
$$

Suppose $f(u)>0$. After (2.4), we have $z(u)<0<w(u)$, because $u \in] 0,1[$. Thus, condition (2.33) boils down to

$$
z(u)=-\frac{f(u)}{1-u} \leq f^{\prime}(u) \leq \frac{f(u)}{u}=w(u) .
$$

This double inequality is equivalent to

$$
-\left[(1-u) f^{\prime}(u)+f(u)\right] \leq 0 \text { and } u f^{\prime}(u)-f(u) \leq 0,
$$

whence $z^{\prime}(u) \leq 0$ and $w^{\prime}(u) \leq 0$. We proceed similarly in the case $f(u)<0$ for which $w(u)<0<z(u)$. If $f(u)=0$, necessarily $w(u)=z(u)=0$ because $u \notin\{0,1\}$. By (2.33), we have $f^{\prime}(u)=0$, from which we deduce that $w^{\prime}(u)=z^{\prime}(u)=0$ via (2.36). To establish the converse, we follow the same steps in reverse order.

It follows that a convex or concave function $f$ with $f(0)=f(1)=0$ is eligible functions all over $[0,1]$. In Lemma 2.2, the function $g$ is hardly visible. We now give it a prominent role in the second test of the Whitham condition. The result below will be useful in $\S 3$.

Lemma 2.3. Let $u \in] 0,1[$. The Whitham condition (2.33) is satisfied at $u$ if and only if

$$
g^{\prime}(u) \in\left\lfloor-\frac{g(u)}{u}, \frac{g(u)}{1-u}\right\rceil .
$$

Proof In terms of $g$, we have

$$
w^{\prime}=-g+(1-u) g^{\prime} \quad \text { and } \quad z^{\prime}=-g-u g^{\prime} .
$$

We apply Lemma 2.2. If $g(u)>0$, then $f(u)>0$ and the decreasing conditions $w^{\prime}(u) \leq 0$ and $z^{\prime}(u) \leq 0$ imply

$$
g(u) \geq \max \left\{(1-u) g^{\prime}(u),-u g^{\prime}(u)\right\} .
$$

Likewise, if $g(u)<0$, we arrive at

$$
g(u) \leq \min \left\{(1-u) g^{\prime}(u),-u g^{\prime}(u)\right\}
$$

From (2.41) and (2.42), we infer (2.39) for $g(u) \neq 0$. If $g(u)=0$, by Lemma 2.2, we must have $w^{\prime}(u)=z^{\prime}(u)=0$, and using $(2.40)$, we have $g^{\prime}(u)=0$. The converse follows the same lines.

\subsection{Riemann problem}

As was explained in $[2,3]$, we need to solve the Riemann problem corresponding to the homogeneous relaxation system in order to know the value of the numerical flux.

A generic point in $\bar{\Omega}$ is designated by $\mathbf{v}=(W, Z)$. From now on, the symbols $v^{-}$and $v^{+}$stand for the negative and positive parts of any real number $v \in \mathbb{R}$. For $(\mathfrak{a}, \mathfrak{b}) \in \mathbb{R}^{2}$, we use the notation

$$
\lfloor\mathfrak{a}, \mathfrak{b}\rceil=\{r \mathfrak{a}+(1-r) \mathfrak{b}, r \in[0,1]\} .
$$

We also denote by $\mathbb{1}_{\{.\}}$the characteristic function. 

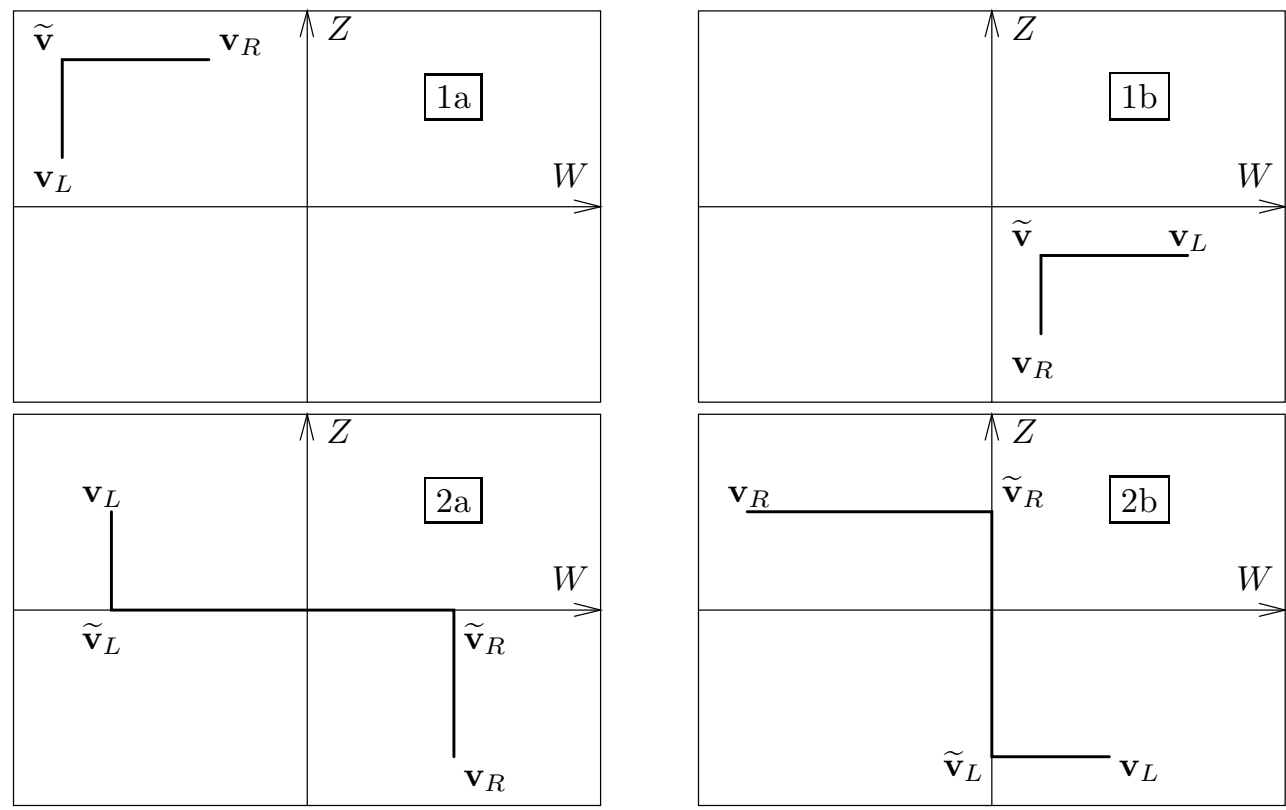

Figure 2: Solution to the Riemann problem for the homogeneous Born-Infeld system.

Theorem 2.4. Let $\mathbf{v}_{L}=\left(W_{L}, Z_{L}\right) \in \bar{\Omega}$ and $\mathbf{v}_{R}=\left(W_{R}, Z_{R}\right) \in \bar{\Omega}$ be two arbitrarily given states. Define the two speeds

$$
s_{L}=\min \left(W_{L}, Z_{L}\right) \leq 0 \quad \text { and } \quad s_{R}=\max \left(W_{R}, Z_{R}\right) \geq 0 .
$$

When $\lambda=0$, the solution to (2.17) for $(t, x) \in \mathbb{R}_{+}^{*} \times \mathbb{R}$ with the initial data

$$
\mathbf{v}(t=0 ; x)=\mathbf{v}_{L} \mathbb{1}_{\{x<0\}}+\mathbf{v}_{R} \mathbb{1}_{\{x>0\}}
$$

is given by

1. If $W_{L} W_{R} \geq 0$ and $Z_{L} Z_{R} \geq 0$,

$$
\mathbf{v}(t, x)=\mathbf{v}_{L} \mathbb{1}_{\left\{x<t s_{L}\right\}}+\widetilde{\mathbf{v}} \mathbb{1}_{\left\{t s_{L}<x<t s_{R}\right\}}+\mathbf{v}_{R} \mathbb{1}_{\left\{x>t s_{R}\right\}}
$$

with $\widetilde{\mathbf{v}}=(\widetilde{W}, \widetilde{Z})=\left(W_{L}^{-}+W_{R}^{+}, Z_{L}^{-}+Z_{R}^{+}\right)$. The intermediate state $\widetilde{\mathbf{v}}$ satisfies the maximum principle

$$
\widetilde{W} \in\left\lfloor W_{L}, W_{R}\right\rceil, \quad \widetilde{Z} \in\left\lfloor Z_{L}, Z_{R}\right\rceil .
$$

2. If $W_{L} W_{R}<0$ or $Z_{L} Z_{R}<0$,

$$
\mathbf{v}(t, x)=\mathbf{v}_{L} \mathbb{1}_{\left\{x<t s_{L}\right\}}+\widetilde{\mathbf{v}}_{L} \mathbb{1}_{\left\{t s_{L}<x<0\right\}}+\widetilde{\mathbf{v}}_{R} \mathbb{1}_{\left\{0<x<t s_{R}\right\}}+\mathbf{v}_{R} \mathbb{1}_{\left\{x>t s_{R}\right\}}
$$

with $\widetilde{\mathbf{v}}_{L}=\left(\widetilde{W}_{L}, \widetilde{Z}_{L}\right)=\left(W_{L}^{-}, Z_{L}^{-}\right)$and $\widetilde{\mathbf{v}}_{R}=\left(\widetilde{W}_{R}, \widetilde{Z}_{R}\right)=\left(W_{R}^{+}, Z_{R}^{+}\right)$. The intermediate states $\widetilde{\mathbf{v}}_{L}$ and $\widetilde{\mathbf{v}}_{R}$ satisfy the maximum principle

$$
\begin{array}{ll}
\widetilde{W}_{L} \in\left\lfloor W_{L}, W_{R}\right\rceil, & \widetilde{Z}_{L} \in\left\lfloor Z_{L}, Z_{R}\right\rceil \\
\widetilde{W}_{R} \in\left\lfloor W_{L}, W_{R}\right\rceil, & \widetilde{Z}_{R} \in\left\lfloor Z_{L}, Z_{R}\right\rceil .
\end{array}
$$


Proof In the $(W, Z)$-plane, $W$-curves are vertical lines, while $Z$-curves are horizontal lines. Riemann problems can thus be solved "by hand," depending on the locations of the two initial states, as depicted in Fig. 2. The claims about the maximum principle are easy to check.

The benefit of the maximum principle (2.47), (2.49) is obvious: should we need the apparent velocities at some intermediate state, we no longer have to worry about the division problem for (1.11), as mentioned in the Introduction. We now concentrate on the flux that is to be plugged in the numerical scheme. In this respect, it is well known $[9,14]$ that in the context of Godunov-like methods, we have to extract the interface values

$$
W^{*}=W\left(x / t=0^{+}\right), \quad Z^{*}=Z\left(x / t=0^{+}\right)
$$

from the full Riemann solution. In the special cases $s_{L}=0$ or $s_{R}=0$, although $W^{*}, Z^{*}$ may take different values at $x / t=0^{+}$and at $x / t=0^{-}$, the relaxed flux

$$
F^{*}=F\left(W^{*}, Z^{*}\right)=\frac{W^{*} Z^{*}}{Z^{*}-W^{*}}
$$

is continuous across $x / t=0$, as detailed by the following procedure.

Theorem 2.5. Let $\mathbf{v}_{L}=\left(W_{L}, Z_{L}\right) \in \bar{\Omega}$ and $\mathbf{v}_{R}=\left(W_{R}, Z_{R}\right) \in \bar{\Omega}$ be two arbitrarily given states.

1. If $W_{L} W_{R} \geq 0$ and $Z_{L} Z_{R} \geq 0$, then the intermediate state $(\widetilde{W}, \widetilde{Z})=\left(W_{L}^{-}+W_{R}^{+}, Z_{L}^{-}+\right.$ $\left.Z_{R}^{+}\right)$, defined in Theorem 2.4, necessarily belongs to $\bar{\Omega}$, and

$$
F^{*}=F(\widetilde{W}, \widetilde{Z}) \text {. }
$$

2. If $W_{L} W_{R}<0$ or $Z_{L} Z_{R}<0$, then $F^{*}=0$.

In all cases, the relaxed flux satisfies the sign property

$$
F^{*} \cdot F\left(W_{L}, Z_{L}\right) \geq 0, \quad F^{*} \cdot F\left(W_{R}, Z_{R}\right) \geq 0 .
$$

Proof The proof is done with the help of Theorem 2.4, the extension (2.13) and by scrutinizing all the situations that can occur. We leave it to the reader.

The sign property (2.53) is a major asset of the Born-Infeld relaxation when it comes to make use of the flux $F^{*}$ for a further purpose. It helps us avoid a bad upwinding in (1.9), as was sketched out in the Introduction and as will be more thoroughly described in $\S 3.3$. To emphasize that $F^{*}$ depends on the left state $\mathbf{v}_{L}$ and the right state $\mathbf{v}_{R}$, we write

$$
F^{*}=\mathscr{F}\left(\mathbf{v}_{L}, \mathbf{v}_{R}\right) .
$$

Definition 2.2. Let $u_{L} \in[0,1]$ and $u_{R} \in[0,1]$ be two arbitrary states. Consider $\mathbf{v}\left(u_{L}\right)=$ $\left(w\left(u_{L}\right), z\left(u_{L}\right)\right)$ and $\mathbf{v}\left(u_{R}\right)=\left(w\left(u_{R}\right), z\left(u_{R}\right)\right)$. Then,

$$
H^{\mathrm{BI}}\left(u_{L}, u_{R}\right)=\mathscr{F}\left(\mathbf{v}\left(u_{L}\right), \mathbf{v}\left(u_{R}\right)\right)
$$

is said to be the Born-Infeld numerical flux associated to $\left(u_{L}, u_{R}\right)$.

Thanks to Theorem 2.5, the Born-Infeld numerical flux is well-defined for all $\left(u_{L}, u_{R}\right) \in$ $[0,1]^{2}$. It is a trivial matter to check that this is a consistant flux, that is, $H^{\mathrm{BI}}(u, u)=f(u)$ for all $u \in[0,1]$. 


\subsection{Stability at the discrete level}

In $\S 2.3$, the Whitham condition was derived and discussed at the continuous level from the Chapman-Enskog analysis. We are going to evidence its connection with the monotonicity of the numerical flux at the discrete level. Consider the first-order explicit scheme

$$
u_{i}^{n+1}=u_{i}^{n}-\frac{\Delta t}{\Delta x}\left[H\left(u_{i}^{n}, u_{i+1}^{n}\right)-H\left(u_{i-1}^{n}, u_{i}^{n}\right)\right] .
$$

We recall [9] that the numerical flux $H$ is said to be monotonous if it is increasing with respect to its first argument and decreasing with respect to its second argument. If we specify $H\left(u_{L}, u_{R}\right)$ for the arguments, then monotonicity is expressed as

$$
H_{u_{L}} \geq 0 \quad \text { and } \quad H_{u_{R}} \leq 0
$$

provided that the partial derivatives exist. We wish to investigate about the monotonicity of the Born-Infeld numerical flux $H^{\mathrm{BI}}\left(u_{L}, u_{R}\right)$ defined in (2.55).

Theorem 2.6. Assume that $g(u)$ keeps a constant sign over $u \in] 0,1[$. Then the BornInfeld numerical flux $H^{\mathrm{BI}}\left(u_{L}, u_{R}\right)$ is monotonous for all $\left.\left(u_{L}, u_{R}\right) \in\right] 0,1[2$ if and only if the Whitham condition (2.33) is satisfied for all $u \in] 0,1[$.

Proof Let us assume $g<0$. Then

$$
\begin{aligned}
& \mathbf{v}_{L}=\left(W_{L}, Z_{L}\right)=\left(w\left(u_{L}\right), z\left(u_{L}\right)\right) \in \mathbb{R}_{-}^{*} \times \mathbb{R}_{+}^{*} \\
& \mathbf{v}_{R}=\left(W_{R}, Z_{R}\right)=\left(w\left(u_{R}\right), z\left(u_{R}\right)\right) \in \mathbb{R}_{-}^{*} \times \mathbb{R}_{+}^{*} .
\end{aligned}
$$

We are in Case $1 \mathrm{a}$ of Fig. 2, which yields $\mathbf{v}^{*}=\widetilde{\mathbf{v}}=\left(W_{L}, Z_{R}\right)$. It follows that

$$
H^{\mathrm{BI}}\left(u_{L}, u_{R}\right)=\frac{w\left(u_{L}\right) z\left(u_{R}\right)}{z\left(u_{R}\right)-w\left(u_{L}\right)}
$$

Thus, the Born-Infeld numerical flux is differentiable and one has

$$
\begin{aligned}
& H_{u_{L}}^{\mathrm{BI}}=w^{\prime}\left(u_{L}\right)\left[\frac{z\left(u_{R}\right)}{z\left(u_{R}\right)-w\left(u_{L}\right)}\right]^{2} \\
& H_{u_{R}}^{\mathrm{BI}}=-z^{\prime}\left(u_{R}\right)\left[\frac{w\left(u_{L}\right)}{z\left(u_{R}\right)-w\left(u_{L}\right)}\right]^{2} .
\end{aligned}
$$

The monotonicity conditions (2.57) take place if and only if $w^{\prime}\left(u_{L}\right) \geq 0$ and $z^{\prime}\left(u_{R}\right) \geq 0$ for all $\left.\left(u_{L}, u_{R}\right) \in\right] 0,1\left[{ }^{2}\right.$. This is equivalent to saying that $w^{\prime}(u) \geq 0$ and $z^{\prime}(u) \geq 0$ for all $u \in] 0,1[$. Since $f(u)<0$, this is still equivalent to the statement that the Whitham condition (2.33) is satisfied at all $u \in] 0,1$ [ by virtue of Lemma 2.2. The case $g>0$ works alike.

As a matter of fact, it is fair to point out that for the Jin-Xin relaxation scheme, the monotonicity of the numerical flux

$$
H^{\mathrm{JX}}\left(u_{L}, u_{R}\right)=\frac{f\left(u_{L}\right)+f\left(u_{R}\right)}{2}-b \frac{u_{R}-u_{L}}{2} \quad \text { for all }\left(u_{L}, u_{R}\right)
$$

is also equivalent to the non-strict Whitham condition

$$
b \geq\left|f^{\prime}(u)\right| \quad \text { for all } u .
$$

In Appendix A, we will show that this equivalence still holds true for a generalized BornInfeld relaxation method. 


\section{Relaxation via Born-Infeld for a Lagrangian two-phase drift-flux model}

Let us return back to the two-phase system (1.1), that we rewrite as

$$
\begin{aligned}
& \partial_{t} \tau-\partial_{m} v=0 \\
& \partial_{t} v+\partial_{m} P(\mathbf{u})=0 \\
& \partial_{t} Y-\partial_{m} \sigma(\mathbf{u})=0
\end{aligned}
$$

with the vector notation

$$
\mathbf{u}:=(\tau, v, Y) \in \mathbb{R}_{+}^{*} \times \mathbb{R} \times[0,1]=: £
$$

and under the assumption

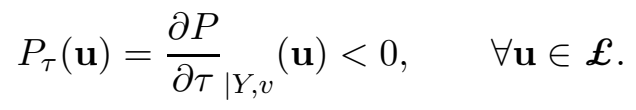

Except for simple slip laws, this assumption is usually not enough to ensure hyperbolicity for (3.1). Since

$$
\sigma(\mathbf{u})=Y(1-Y) \rho \phi(\mathbf{u})
$$

we introduce

$$
w(\mathbf{u})=-\frac{\sigma(\mathbf{u})}{Y}=-(1-Y) \rho \phi(\mathbf{u}), \quad z(\mathbf{u})=\frac{\sigma(\mathbf{u})}{1-Y}=Y \rho \phi(\mathbf{u})
$$

by analogy with (2.4). These can be interpreted as Lagrangian convective velocities for the phases, to the extent that

$$
\partial_{t}(Y)+\partial_{m}(Y w(\mathbf{u}))=0, \quad \text { and } \quad \partial_{t}(1-Y)+\partial_{m}((1-Y) z(\mathbf{u}))=0 .
$$

The difference

$$
g(\mathbf{u})=w(\mathbf{u})-z(\mathbf{u})=-\rho \phi(\mathbf{u})
$$

then appears to be the Lagrangian slip velocity between the gas $(Y)$ and the liquid $(1-Y)$. The transformations (3.5) can be inverted to yield

$$
Y=\frac{z(\mathbf{u})}{z(\mathbf{u})-w(\mathbf{u})}, \quad-\sigma=\frac{w(\mathbf{u}) z(\mathbf{u})}{z(\mathbf{u})-w(\mathbf{u})}
$$

provided that $w(\mathbf{u}) \neq z(\mathbf{u})$.

\subsection{Relaxation system}

The basic idea is to apply the Born-Infeld relaxation of $\S 2$ to the single equation (3.1c), while keeping the same relaxation technique as $[2,3]$ for $P(\mathbf{u})$ in the acoustic block. To express this more clearly, we need a few notations.

As in $\S 2$, we consider the pair $(W, Z) \in \bar{\Omega}$ to be the relaxation counterparts of the equilibrium values $(w(\mathbf{u}), z(\mathbf{u}))$. In imitation of (3.8), we define

$$
\Upsilon(W, Z)=\frac{Z}{Z-W}, \quad-\Sigma(W, Z)=\frac{W Z}{Z-W}
$$


to be the relaxation counterparts of $(Y,-\sigma(\mathbf{u}))$, with the implicit identification $\Upsilon \equiv Y \in$ $[0,1]$. The inverse transformation of $(3.9)$ is

$$
W(\Upsilon, \Sigma)=-\frac{\Sigma}{\Upsilon}, \quad Z(\Upsilon, \Sigma)=\frac{\Sigma}{1-\Upsilon}
$$

Define

$$
G=W-Z
$$

Then,

$$
-\Sigma=\Upsilon(1-\Upsilon) G, \quad W=(1-\Upsilon) G, \quad Z=-\Upsilon G
$$

Finally, the relaxation version of the vector notation (3.2) is

$$
\mathbf{U}=(\tau, v, \Upsilon(W, Z)) \in £ .
$$

The relaxation strategy we propose for (3.1) consists in considering the system

$$
\begin{aligned}
\partial_{t} \tau-\partial_{m} v & =0 \\
\partial_{t} v+\partial_{m} \Pi & =0 \\
\partial_{t} \Pi+a^{2} \partial_{m} v & =\lambda(P(\mathbf{U})-\Pi) \\
\partial_{t} W+Z \partial_{m} W & =\lambda(w(\mathbf{U})-W) \\
\partial_{t} Z+W \partial_{m} Z & =\lambda(z(\mathbf{U})-Z)
\end{aligned}
$$

for $\lambda>0$, in which $(\tau, v, \Pi, W, Z)$ are considered as independent variables. Unlike (3.1), the homogeneous case $\lambda=0$ of system (3.14) never fails to be hyperbolic, provided that $a \neq 0$. Its (unordered) eigenvalues

$$
-a, W, 0, Z,+a,
$$

associated with the strong Riemann invariants

$$
\Pi-a v, Z, \Pi+a^{2} \tau, W, \Pi+a v
$$

are all linearly degenerate. This allows us to derive the equivalent form

$$
\begin{aligned}
\partial_{t} \tau-\partial_{m} v & =0 \\
\partial_{t} v+\partial_{m} \Pi & =0 \\
\partial_{t} \Pi+a^{2} \partial_{m} v & =\lambda(P(\mathbf{u})-\Pi) \\
\partial_{t} Y+\partial_{m} Y(1-Y) G & =0 \\
\partial_{t} G+G^{2} \partial_{m} Y & =\lambda(g(\mathbf{u})-G),
\end{aligned}
$$

along the same lines as in $\S 2$.

Should the multi-component conservation laws

$$
\begin{aligned}
\partial_{t}(Y \xi)-\partial_{m}(\xi \sigma) & =0 \\
\partial_{t}((1-Y) \eta)+\partial_{m}(\eta \sigma) & =0
\end{aligned}
$$

be appended to system $(3.1)$, in which case $(\xi, \eta)$ must also be considered as inputs for $(P, \sigma)$, we would attach the subsystem

$$
\begin{aligned}
\partial_{t}(\Upsilon \xi)-\partial_{m}(\xi \Sigma) & =0 \\
\partial_{t}((1-\Upsilon) \eta)+\partial_{m}(\eta \Sigma) & =0
\end{aligned}
$$

to the relaxation system (3.14), where $\Upsilon(W, Z)$ and $\Sigma(W, Z)$ are given by (3.9). 


\subsection{Whitham conditions and closure laws}

The essence of the relaxation technique advocated in [3] is to envision the acoustic block (3.14a) $-(3.14 \mathrm{c})$ as separated from the kinematic block $(3.14 \mathrm{~d})-(3.14 \mathrm{e})$. This is achieved by setting $\lambda=0$ and to solve the corresponding Riemann problem. But this decoupling paradigm goes even further. It was justified at length in [3] that in the Chapman-Enskog analysis for $\lambda \rightarrow \infty$, it is still legitimate to see the two blocks as independent from each other. As far as stability is concerned, this argument leads to the Whitham conditions

$$
\begin{aligned}
a & \geq\left\{-P_{\tau}+P_{v}^{2}\right\}(\mathbf{u}) \\
-\sigma_{Y}(\mathbf{u}) & \in\lfloor w(\mathbf{u}), z(\mathbf{u})\rceil
\end{aligned}
$$

for all $\mathbf{u}$ under consideration. In $(3.20 \mathrm{~b})$, the symbol $\sigma_{Y}$ stands for the partial derivative

$$
\sigma_{Y}=\frac{\partial \sigma}{\partial Y}{ }_{\mid \tau, v}
$$

Observe that condition (3.20b) is none other than inequality (2.33) of Theorem 2.3 for $f \equiv-\sigma$ and $u \equiv Y$. For the stability of system (3.19a), this is a heuristic requirement.

Since $\sigma=Y(1-Y) \phi$, we apply Lemma 2.3 to characterize the Whitham condition (3.20b) in terms of $\phi$ alone. This gives rise to

$$
\phi_{Y}(\mathbf{u}) \in\left\lfloor-\frac{\phi(\mathbf{u})}{Y}, \frac{\phi(\mathbf{u})}{1-Y}\right\rceil .
$$

The miracle is that most of the slip laws commonly used by physicists pass the test (3.22)! To name a few:

- The dispersed law for small bubbles of gas in the liquid

$$
\phi=\frac{V_{\infty} \tau}{1-Y}
$$

for a given $V_{\infty} \in \mathbb{R}$. This law is not valid for $Y \rightarrow 1$.

- The Zuber-Findlay law $[4,20]$ for intermittent regimes

$$
\phi=\frac{\left(C_{0}-1\right) v+C_{1}}{(1-Y)\left[1-C_{0}\left(1-\tau_{\ell}^{0} / \tau\right)\right]}
$$

where $C_{0}>1, C_{1} \in \mathbb{R}$ and $\tau_{\ell}^{0}>0$ are given constants. This law is not valid for $Y \rightarrow 1$ either.

- The modified Zuber-Findlay law

$$
\phi=-\frac{\mu v+\nu}{1-\mu(1-Y)\left(1-\tau_{\ell}^{0} / \tau\right)}
$$

where $\mu \in] 0,1\left[, \nu \in \mathbb{R}\right.$ and $\tau_{\ell}^{0}>0$ are given constants. This law is valid for all $Y \in[0,1]$.

The actual verification follows from straightforward calculations that we leave to the reader. As revealed by the calculations, $\phi_{Y}$ covers the whole interval in the right-hand side of (3.22), which means that the Whitham bounds are "optimal." 


\subsection{Explicit scheme}

In the context of oil and gas transportation along a pipeline, the situation that is of interest to engineers is, undoubtedly, subsonic flow with low Mach numbers. Since the relaxation parameter $a$ is comparable to the Lagrangian speed of acoustic waves, we typically have

$$
a \gg \max \{|w(\mathbf{u})|,|z(\mathbf{u})|\} .
$$

At the discrete level, let us consider a uniform mesh of size $\Delta m$ and a time-step $\Delta t$. Since $\pm a$ are the dominant eigenvalues of the relaxation system (3.14), we have to respect the CFL condition

$$
\frac{a \Delta t}{\Delta m}<1
$$

where $a$ itself abides by a discrete version of the Whitham condition (3.20a), namely,

$$
a=\max _{j \in \mathbb{Z}}\left\{-P_{\tau}+P_{v}^{2}\right\}\left(\mathbf{u}_{j}^{n}\right)
$$

In (3.28), the subscript $j$ denotes the cell index and the superscript $n$ the current timelevel. Within the framework of a first-order explicit schemes, the update formulae for (3.1) using the relaxation method (3.14) are

$$
\begin{aligned}
& \frac{\tau_{j}^{n+1}-\tau_{j}^{n}}{\Delta t}-\frac{v_{j+1 / 2}^{*}-v_{j-1 / 2}^{*}}{\Delta m}=0 \\
& \frac{v_{j}^{n+1}-v_{j}^{n}}{\Delta t}+\frac{\Pi_{j+1 / 2}^{*}-\Pi_{j-1 / 2}^{*}}{\Delta m}=0 \\
& \frac{Y_{j}^{n+1}-Y_{j}^{n}}{\Delta t}-\frac{\Sigma_{j+1 / 2}^{*}-\Sigma_{j-1 / 2}^{*}}{\Delta m}=0,
\end{aligned}
$$

with

$$
\begin{aligned}
v_{j+1 / 2}^{*} & =\frac{v_{j}^{n}+v_{j+1}^{n}}{2}-\frac{P_{j+1}^{n}-P_{j}^{n}}{2 a} \\
\Pi_{j+1 / 2}^{*} & =\frac{P_{j}^{n}+P_{j+1}^{n}}{2}-a \frac{v_{j+1}^{n}-v_{j}^{n}}{2}
\end{aligned}
$$

and

$$
-\Sigma_{j+1 / 2}^{*}=\mathscr{F}\left(\mathbf{v}_{j}^{n}, \mathbf{v}_{j+1}^{n}\right) .
$$

In (3.30), $P_{j}^{n}=P\left(\mathbf{u}_{j}^{n}\right)$. As for the notation $\mathscr{F}\left(\mathbf{v}_{L}, \mathbf{v}_{R}\right)$ for the Godunov flux as a function of the left and right initial velocities $\mathbf{v}=(W, Z)$ in (3.31), it was defined in $\S 2$, by equation (2.54). Here, the input arguments are

$$
\mathbf{v}_{j}^{n}=\left(w\left(\mathbf{u}_{j}^{n}\right), z\left(\mathbf{u}_{j}^{n}\right)\right), \quad \mathbf{v}_{j+1}^{n}=\left(w\left(\mathbf{u}_{j+1}^{n}\right), z\left(\mathbf{u}_{j+1}^{n}\right)\right) .
$$

Had we applied Jin-Xin's relaxation

$$
\begin{aligned}
& \partial_{t} Y-\partial_{m} \Sigma=0 \\
& \partial_{t} \Sigma-b^{2} \partial_{m} Y=\lambda(\sigma(\mathbf{u})-\Sigma),
\end{aligned}
$$

to the "scalar" equation (3.1c) with a Whitham-complying choice for $b$, e.g.,

$$
b=\max _{j \in \mathbb{Z}}\left|\sigma_{Y}\left(\mathbf{u}_{j}^{n}\right)\right|
$$


the update formulae would have been the same as (3.29)-(3.30), but with

$$
\Sigma_{j+1 / 2}^{*}=\frac{\sigma_{j}^{n}+\sigma_{j+1}^{n}}{2}+b \frac{Y_{j+1}^{n}-Y_{j}^{n}}{2} .
$$

using the short-hand notation $\sigma_{i}^{n}=\sigma\left(\mathbf{u}_{i}^{n}\right)$. As a result, $\Sigma_{i+1 / 2}^{*}$ may have a sign opposite to that of the $\sigma$ 's if $b$ is large. More accurately, for

$$
b>\left|\frac{\sigma_{j+1}^{n}-\sigma_{j}^{n}}{Y_{j+1}^{n}-Y_{j}^{n}}\right|,
$$

which is not so large a bound, for this is the discrete version of $b>\left|\sigma_{Y}\right|$, we have

$$
\Sigma_{j+1 / 2}^{*} \notin\left\lfloor\sigma_{j}^{n}, \sigma_{j+1}^{n}\right\rceil .
$$

Thus, $\Sigma_{j+1 / 2}^{*}$ can be positive while $\sigma_{j}^{n}$ and $\sigma_{j+1}^{n}$ are both negative, or vice-versa. Therefore, in the discretization

$$
\begin{aligned}
\frac{(Y \xi)_{j}^{n+1}-(Y \xi)_{j}^{n}}{\Delta t}-\frac{(\xi \Sigma)_{j+1 / 2}^{*}-(\xi \Sigma)_{j-1 / 2}^{*}}{\Delta m} & =0 \\
\frac{((1-Y) \eta)_{j}^{n+1}-((1-Y) \xi)_{j}^{n}}{\Delta t}+\frac{(\eta \Sigma)_{j+1 / 2}^{*}-(\eta \Sigma)_{j-1 / 2}^{*}}{\Delta m} & =0
\end{aligned}
$$

of (3.19a), the upwinded values

$$
\left(\xi_{j+1 / 2}^{*}, \eta_{j+1 / 2}^{*}\right)= \begin{cases}\left(\xi_{j}^{n}, \eta_{j+1}^{n}\right) & \text { if } \Sigma_{j+1 / 2}^{*}<0 \\ \left(\xi_{j+1}^{n}, \eta_{j}^{n}\right) & \text { if } \Sigma_{j+1 / 2}^{*}>0\end{cases}
$$

are likely to come from the "wrong" side. Although this cannot be blamed for creating instabilities, it induces more dissipation.

As announced in the Introduction, the real drawback of (3.33) turns up when we need to know the velocities

$$
\frac{\Sigma_{j+1 / 2}^{*}}{Y_{j+1 / 2}^{*}} \text { and } \frac{\Sigma_{j+1 / 2}^{*}}{1-Y_{j+1 / 2}^{*}}
$$

corresponding to the intermediate state, with

$$
Y_{j+1 / 2}^{*}=\frac{Y_{j}^{n}+Y_{j+1}^{n}}{2}+\frac{\sigma_{j+1}^{n}-\sigma_{j}^{n}}{2 b} .
$$

Indeed, the values (3.40) are the speeds of waves advecting $\xi$ and $\eta$ in the Riemann problem at edge $j+1 / 2$. We have to compute them in order to construct an equivalent Roe-matrix that would enable us to extend the scheme to an implicit or hybrid explicit-implicit timeintegration [3]. In the neighborhood of single-phase states, i.e., $Y=0$ or 1 , the practical implementation of (3.40) is flawed with division-by-zero problems, the handling of which is awesome. The advantage of the Born-Infeld relaxation is that we do not have to compute the velocities of the intermediate state by a risky floating operation. 


\section{Relaxation via Born-Infeld for an Eulerian two-phase drift- flux model}

The Eulerian formulation of the two-phase model (3.1) is

$$
\begin{aligned}
& \partial_{t}(\rho)+\partial_{x}(\rho v)=0 \\
& \partial_{t}(\rho v)+\partial_{x}\left(\rho v^{2}+P(\mathbf{q})\right)=0 \\
& \partial_{t}(\rho Y)+\partial_{x}(\rho Y v-\sigma(\mathbf{q}))=0,
\end{aligned}
$$

with the vector notation

$$
\mathbf{q} \in \boldsymbol{\epsilon}=\{(\rho, \rho v, \rho Y) \mid \rho>0, v \in \mathbb{R}, Y \in[0,1]\} .
$$

On the grounds of practicalities, we use the same letters $P, \sigma$ to designate functions of the Eulerian variables $\mathbf{q} \in \boldsymbol{E}$ in (4.1) and functions of the Lagrangian variables $\mathbf{u} \in £$ in (3.1). We recall that $\rho=\tau^{-1}$ is the density. We impose the same condition (3.3) on the total pressure $P$, without the guarantee of hyperbolicity except for the usual slip laws.

\subsection{Relaxation system}

We introduce $(W, Z) \in \bar{\Omega}$ in the same fashion as in $\S 3$. For convenience, we denote the relaxation counterpart of $\mathbf{q}$ by

$$
\mathbf{Q}=(\rho, \rho v, \rho \Upsilon(W, Z)),
$$

in which $\Upsilon$ is computed by (3.9). Following the philosophy of $[2,3]$, we decide that the relaxation system for (4.1) is the Eulerian transform of (3.14), which yields

$$
\begin{aligned}
\partial_{t}(\rho)+\partial_{x}(\rho v) & =0 \\
\partial_{t}(\rho v)+\partial_{x}\left(\rho v^{2}+\Pi\right) & =0 \\
\partial_{t}(\rho \Pi)+\partial_{x}\left(\rho \Pi v+a^{2} v\right) & =\lambda \rho(P(\mathbf{Q})-\Pi) \\
\partial_{t}(\rho W)+\partial_{x}(\rho W v)+Z \partial_{x} W & =\lambda \rho(w(\mathbf{Q})-W) \\
\partial_{t}(\rho Z)+\partial_{x}(\rho Z v)+W \partial_{x} Z & =\lambda \rho(z(\mathbf{Q})-Z)
\end{aligned}
$$

The last two equations can be recombined to give

$$
\begin{aligned}
& \partial_{t} W+(v+Z \tau) \partial_{x} W=\lambda(w(\mathbf{Q})-W) \\
& \partial_{t} Z+(v+W \tau) \partial_{x} Z=\lambda(z(\mathbf{Q})-Z) .
\end{aligned}
$$

It is easy to check that the relaxation system (4.4) is always hyperbolic. Its (unordered) eigenvalues

$$
v-a \tau, v+W \tau, v, v+Z \tau, v+a \tau,
$$

associated with the Riemann invariants (3.16), are all linearly degenerate. This allows us to derive the equivalent form

$$
\begin{aligned}
\partial_{t}(\rho)+\partial_{x}(\rho v) & =0 \\
\partial_{t}(\rho v)+\partial_{x}\left(\rho v^{2}+\Pi\right) & =0 \\
\partial_{t}(\rho \Pi)+\partial_{x}\left(\rho \Pi v+a^{2} v\right) & =\lambda \rho(P(\mathbf{q})-\Pi) \\
\partial_{t}(\rho Y)+\partial_{x}(\rho Y v+Y(1-Y) G) & =0 \\
\partial_{t}(\rho G)+\partial_{x}(\rho G v)+G^{2} \partial_{x} Y & =\lambda \rho(g(\mathbf{q})-G)
\end{aligned}
$$


by standard calculations, with $g(\mathbf{q})=-\rho Y(1-Y) \phi(\mathbf{q})$.

Should the multi-component conservation laws

$$
\begin{aligned}
\partial_{t}(\rho Y \xi)+\partial_{x}(\xi(\rho Y v-\sigma)) & =0 \\
\partial_{t}(\rho(1-Y) \eta)+\partial_{x}(\eta(\rho(1-Y) v+\sigma)) & =0
\end{aligned}
$$

for some component be appended to system (4.1), in which case $(\xi, \eta)$ must also be considered as inputs for $(P, \sigma)$, we would attach the subsystem

$$
\begin{aligned}
& \partial_{t}(\rho \Upsilon \xi)+\partial_{x}(\xi(\rho \Upsilon v-\Sigma))=0 \\
& \partial_{t}(\rho(1-\Upsilon) \eta)+\partial_{x}(\eta(\rho(1-\Upsilon) v+\Sigma))=0
\end{aligned}
$$

to (4.4), where $\Upsilon(W, Z)$ and $\Sigma(W, Z)$ are given by (3.9).

The Whitham conditions are those of (3.20). The typical low-Mach flow that is of interest to us is now expressed in terms of Eulerian velocities as

$$
|a| \gg \max \{|w(\mathbf{q})|,|z(\mathbf{q})|,|\rho v|\}
$$

which implies $v-a \tau<0$ and $v+a \tau>0$.

\subsection{Explicit scheme}

At the discrete level, let us consider a uniform mesh of size $\Delta x$ and a time-step $\Delta t$. Since $v \pm a \tau$ are the dominant eigenvalues of the relaxation system (4.4), we have to impose the CFL restriction

$$
\frac{\Delta t}{\Delta x} \max _{i \in \mathbb{Z}}\left|v_{i}^{n} \pm a \tau_{i}^{n}\right|<1,
$$

where $a$ is given by (3.28). The subscript $i$ denotes the cell index and the superscript $n$ the current time-level. Within the framework of a first-order explicit schemes, the update formulae for (4.1) using the relaxation method (4.4) are

$$
\begin{aligned}
\frac{(\rho)_{i}^{n+1}-(\rho)_{i}^{n}}{\Delta t}+\frac{(\rho v)_{i+1 / 2}^{\downarrow}-(\rho v)_{i-1 / 2}^{\downarrow}}{\Delta x} & =0 \\
\frac{(\rho v)_{i}^{n+1}-(\rho v)_{i}^{n}}{\Delta t}+\frac{\left(\rho v^{2}+\Pi\right)_{i+1 / 2}^{\downarrow}-\left(\rho v^{2}+\Pi\right)_{i-1 / 2}^{\downarrow}}{\Delta x} & =0 \\
\frac{(\rho Y)_{i}^{n+1}-(\rho Y)_{i}^{n}}{\Delta t}+\frac{(\rho Y v-\Sigma)_{i+1 / 2}^{\downarrow}-(\rho Y v-\Sigma)_{i-1 / 2}^{\downarrow}}{\Delta x} & =0 .
\end{aligned}
$$

Contrary to the Lagrangian case, the Godunov-type values denoted by $(.)_{i+1 / 2}^{\downarrow}$ do not necessarily coincide with the intermediate state $(.)_{i+1 / 2}^{*}$ of the homogeneous Born-Infeld subproblem (4.5) at edge $i+1 / 2$.

For convenience, let us switch to the notation using subscripts $L$ and $R$ for the left and right states of the Riemann problem. After Theorem 2.4, introduce

$$
s_{L}=\min \left(w\left(\mathbf{q}_{L}\right), z\left(\mathbf{q}_{L}\right)\right) \leq 0 \quad \text { and } \quad s_{R}=\max \left(w\left(\mathbf{q}_{R}\right), z\left(\mathbf{q}_{R}\right)\right) \geq 0 .
$$

Under assumption (4.10), the eigenvalues involved in the Riemann problem associated to (4.4) are increasingly ordered as

$$
v_{L}-a \tau_{L}<v^{*}+s_{L} \tau_{L}^{*} \leq v^{*} \leq v^{*}+s_{R} \tau_{R}^{*}<v_{R}+a \tau_{R}
$$


where (see [1-3] for details)

$$
\begin{aligned}
& \tau_{L}^{*}=\tau_{L}+\frac{v_{R}-v_{L}}{2 a}-\frac{P_{R}-P_{L}}{2 a^{2}} \\
& \tau_{R}^{*}=\tau_{R}+\frac{v_{R}-v_{L}}{2 a}+\frac{P_{R}-P_{L}}{2 a^{2}}
\end{aligned}
$$

and

$$
\begin{gathered}
v^{*}=\frac{v_{L}+v_{R}}{2}-\frac{P_{R}-P_{L}}{2 a} \\
\Pi^{*}=\frac{P_{L}+P_{R}}{2}-a \frac{v_{R}-v_{L}}{2},
\end{gathered}
$$

with a clear separation of the orders of magnitude between the speeds

$$
\min \left\{\left|v_{L}-a \tau_{L}\right|,\left|v_{L}+a \tau_{L}\right|\right\} \gg \max \left\{\left|v^{*}+s_{L} \tau_{L}^{*}\right|,\left|v^{*}+s_{R} \tau_{R}^{*}\right|\right\} .
$$

Since $v_{L}-a \tau_{L}<0$ and $v_{R}+a \tau_{R}>0$, we always have

$$
v^{\downarrow}=v^{*}, \quad \Pi^{\downarrow}=\Pi^{*} .
$$

As for $\tau^{\downarrow}$ and $\Sigma^{\downarrow}$, distinction has to be made between several cases depending on the signs of $v^{*}+s_{L} \tau_{L}^{*}, v^{*}$ and $v^{*}+s_{R} \tau_{R}^{*}$. Without entering a tedious discussion including all equality cases, we claim that

$$
\begin{aligned}
\tau^{\downarrow} & =\tau_{L} \mathbb{1}_{\left\{v^{*}+s_{L} \tau_{L}^{*}>0\right\}}+\tau_{L}^{*} \mathbb{1}_{\left\{v^{*}+s_{L} \tau_{L}^{*}<0<v^{*}\right\}} \\
& +\tau_{R}^{*} \mathbb{1}_{\left\{v^{*}<0<v^{*}+s_{R} \tau_{R}^{*}\right\}}+\tau_{R} \mathbb{1}_{\left\{v^{*}+s_{R} \tau_{R}^{*}<0\right\}} \\
\Sigma^{\downarrow} & =\sigma_{L} \mathbb{1}_{\left\{v^{*}+s_{L} \tau_{L}^{*}>0\right\}}-\mathscr{F}\left(\mathbf{v}_{L}, \mathbf{v}_{R}\right) \mathbb{1}_{\left\{\left(v^{*}+s_{L} \tau^{*}\right)\left(v^{*}+s_{R} \tau_{R}^{*}\right)<0\right\}} \\
& +\sigma_{R} \mathbb{1}_{\left\{v^{*}+s_{R} \tau_{R}^{*}<0\right\}}
\end{aligned}
$$

with $\mathbf{v}_{L}=\left(w\left(\mathbf{q}_{L}\right), z\left(\mathbf{q}_{L}\right)\right)$ and $\mathbf{v}_{R}=\left(w\left(\mathbf{q}_{R}\right), z\left(\mathbf{q}_{R}\right)\right)$. The computation of $Y^{\downarrow}$ is lengthier to explain. In case 1 of Theorem 2.4, we write

$$
Y^{\downarrow}=Y_{L} \mathbb{1}_{\left\{v^{*}+s_{L} \tau_{L}^{*}>0\right\}}+U(\widetilde{\mathbf{v}}) \mathbb{1}_{\left\{\left(v^{*}+s_{L} \tau_{L}^{*}\right)\left(v^{*}+s_{R} \tau_{R}^{*}\right)<0\right\}}+Y_{R} \mathbb{1}_{\left\{v^{*}+s_{R} \tau_{R}^{*}<0\right\}},
$$

and in case 2, we write

$$
\begin{aligned}
Y^{\downarrow} & =Y_{L} \mathbb{1}_{\left\{v^{*}+s_{L} \tau_{L}^{*}>0\right\}}+U\left(\widetilde{\mathbf{v}}_{L}\right) \mathbb{1}_{\left\{v^{*}+s_{L} \tau_{L}^{*}<0<v^{*}\right\}} \\
& +U\left(\widetilde{\mathbf{v}}_{R}\right) \mathbb{1}_{\left\{v^{*}<0<v^{*}+s_{R} \tau_{R}^{*}\right\}}+Y_{R} \mathbb{1}_{\left\{v^{*}+s_{R} \tau_{R}^{*}<0\right\}}
\end{aligned}
$$

whenever it makes sense to compute $U($.$) by (2.7)$, that is, for $\widetilde{\mathbf{v}} \neq(0,0)$ or $\widetilde{\mathbf{v}}_{L, R} \neq(0,0)$. It happens that in all situations where $U($.$) is not well-defined, we do not really need Y^{\downarrow}$, thanks either to $\left(v^{*}+s_{L} \tau_{L}^{*}\right)\left(v^{*}+s_{R} \tau_{R}^{*}\right)>0$ or to $v^{\downarrow}=0$.

\subsection{Numerical results}

We compare the numerical solution computed with the Born-Infeld relaxation to that of the relaxation introduced in [2]. All the runs are performed over a 100m-long pipeline, discretized by cells of size $0.5 \mathrm{~m}$. The experiments involve Riemann problems with various types of slip law, for which the original system (4.1) is hyperbolic and admits 3 eigenvalues

$$
\lambda_{1}(\mathbf{q})<\lambda_{2}(\mathbf{q})<\lambda_{3}(\mathbf{q}), \quad \text { with }\left|\lambda_{2}(\mathbf{q})\right| \ll\left|\lambda_{1}(\mathbf{q})\right| \approx\left|\lambda_{3}(\mathbf{q})\right| .
$$




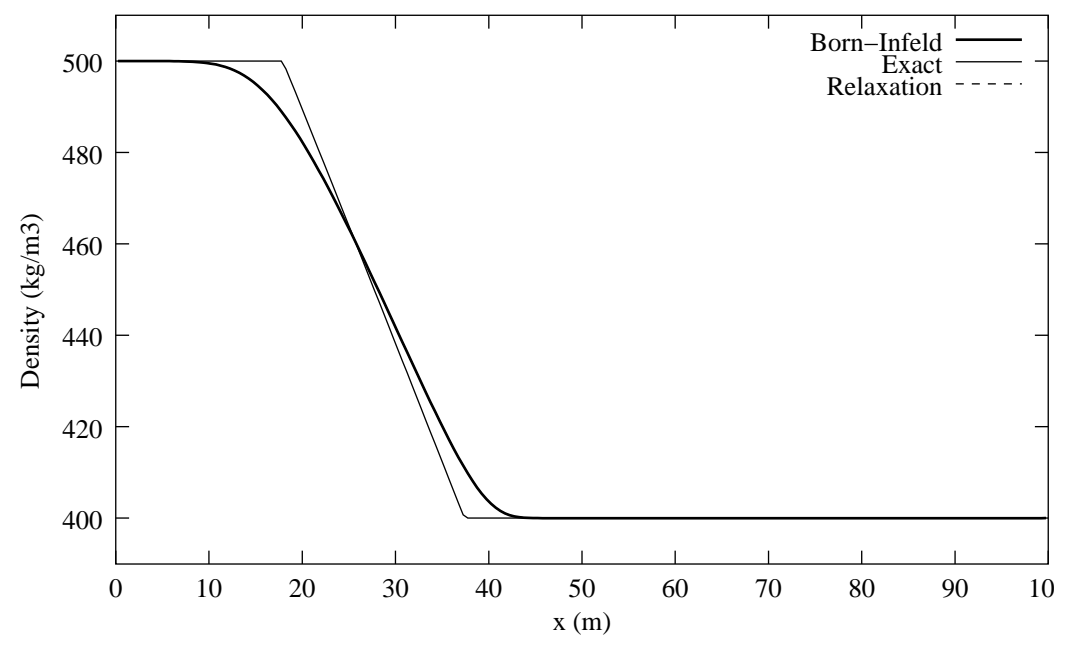

Figure 3: Experiment 1

As for the pressure law, we assume an incompressible liquide $\rho_{\ell}(p)=\rho_{\ell}^{0}$ and a perfect gas $\rho_{g}(p)=p / a_{g}^{2}$, which leads to

$$
p(\rho, \rho Y)=\frac{a_{g}^{2} \rho Y}{1-\tau_{\ell}^{0} \rho(1-Y)}
$$

by virtue of the identity

$$
\frac{\rho Y}{\rho_{g}(p)}+\frac{\rho(1-Y)}{\rho_{\ell}(p)}=1
$$

Unless otherwise indicated, we use $\tau_{\ell}^{0}=10^{-3} \mathrm{~m}^{3} / \mathrm{kg}$ and $a_{g}=300 \mathrm{~m} / \mathrm{s}$.

\subsubsection{Experiment 1}

The simplest slip law to begin with is $\phi \equiv 0$, which means that the gas is moving at the same speed $v$ as the liquid. In this experiment, $a_{g}=100 \mathrm{~m} / \mathrm{s}$. Consider the left $L$ and right $R$ states

$$
\left(\begin{array}{c}
\rho \\
Y \\
v
\end{array}\right)_{L}=\left(\begin{array}{c}
500 \\
0.2 \\
34.423
\end{array}\right) \text { and }\left(\begin{array}{c}
\rho \\
Y \\
v
\end{array}\right)_{R}=\left(\begin{array}{c}
400 \\
0.2 \\
50
\end{array}\right)
$$

These have been tailored so that the solution to the Riemann problem is a pure 1rarefaction, the front of which moves at the speed $\left(\lambda_{1}\right)_{L}=-40.12 \mathrm{~m} / \mathrm{s}$ and the tail of which moves at the speed $\left(\lambda_{1}\right)_{R}=-15.77 \mathrm{~m} / \mathrm{s}$. The snapshot in Fig. 3 corresponds to time $T=0.8 \mathrm{~s}$.

We see that the two approximate solutions are correct and roughly identical. This suggests that the numerical dissipation introduced by the two schemes is the same. This conclusion is confirmed by the next two experiments. 


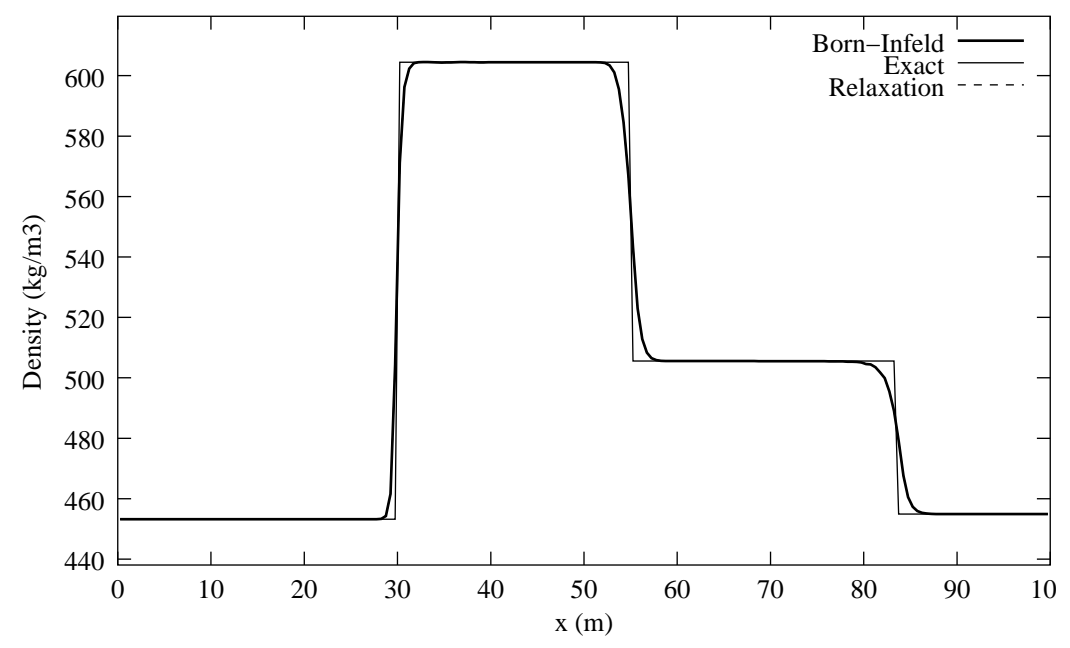

Figure 4: Experiment 2

\subsubsection{Experiment 2}

The second slip law we consider is the Zuber-Findaly law (3.24), with $C_{0}=1.07$ and $C_{1}=0.21620$. The initial left and right states are

$$
\left(\begin{array}{c}
\rho \\
Y \\
v
\end{array}\right)_{L}=\left(\begin{array}{c}
453.19 \\
0.70543 .10^{-2} \\
24.807
\end{array}\right) \quad \text { and } \quad\left(\begin{array}{c}
\rho \\
Y \\
v
\end{array}\right)_{R}=\left(\begin{array}{c}
454.91 \\
0.10804 .10^{-1} \\
1.7460
\end{array}\right)
$$

These have been tailored so that the solution to the Riemann problem is made up of a 1-shock, a 2-contact discontinuity and a 3-shock. The result is shown in Fig. 4, which corresponds to the final time $T=0.5 \mathrm{~s}$. Again, the two schemes exhibit the same behavior.

\subsubsection{Experiment 3}

The slip is now the dispersed law (3.23) with $V_{\infty}=-4480.9$. The initial left and right states are

$$
\left(\begin{array}{c}
\rho \\
Y \\
v
\end{array}\right)_{L}=\left(\begin{array}{c}
901.11 \\
1.2330 .10^{-3} \\
0.95027
\end{array}\right) \text { and }\left(\begin{array}{l}
\rho \\
Y \\
v
\end{array}\right)_{R}=\left(\begin{array}{c}
208.88 \\
4.2552 .10^{-2} \\
0.78548
\end{array}\right)
$$

These have been tailored so that the solution to the Riemann problem is a 2-contact discontinuity propagating at speed $1 \mathrm{~m} / \mathrm{s}$. The result is shown in Fig. 4 at time $T=20 \mathrm{~s}$.

This experience with a non-zero slip law and a slow contact discontinuity is usually stiff enough to rank the schemes. Here, this is not the case and the two relaxation schemes behave very similarly.

\section{Conclusion}

As evidenced throughout this contribution, there is a close connection between scalar conservation laws of the type (2.2) and the Born-Infeld equations (2.17). Insofar as the 


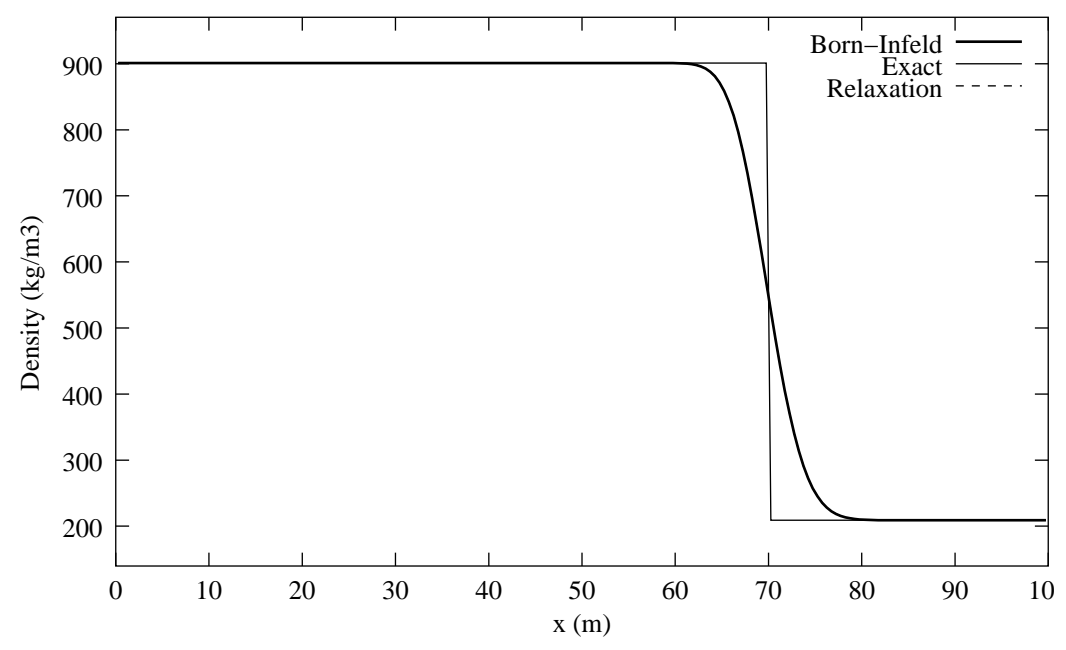

Figure 5: Experiment 3

relaxation method we propose takes advantage of this connection, it looks somehow more natural than the standard Jin-Xin relaxation. For one, there is no need for tuning an extra parameter in order to ensure stability. For another, the eigenvalues of the relaxation system coincide with the physically meaningful phase velocities associated to the original equation.

Besides the aesthetic appeal of the calculations presented in $\S 2$ (see Appendix A for more results in the same vein), the Born-Infeld relaxation method is all the more attractive that, in terms of numerical quality, it does compete well with the Jin-Xin method in which the least diffusion amount is set. For practitioners, the very fact that no free parameter is required might be regarded as a drawback, for there is no "screwdriver" at our disposal to force diffusion when the flux does not belong to the class of eligible functions. However, this class of eligible functions is not as narrow as one might think. It comes as a mystical surprise that, in the area of drift-flux model for two-phase flows, the Whitham condition is met by the most commonly used slip laws!

Once integrated into a full computational process for two-phase flow systems via the decoupling paradigm of [3], the Born-Infeld scalar relaxation helps us solve the tricky issue related to the the phase velocities of the intermediate state, as well as the less tricky, albeit unpleasant issue of the sign of the numerical flux. This makes it easier to upgrade the method to a hybrid explicit-implicit time integration. Numerical experiments show that the overall relaxation scheme behaves well compared to the former one based on the Jin-Xin relaxation.

\section{References}

[1] M. BAudin, Méthodes de relaxation pour la simulation des écoulements polyphasiques dans les conduites pétrolières, Thèse de doctorat, Université Pierre et Marie Curie, 2003.

[2] M. Baudin, C. Berthon, F. Coquel, R. Masson, and Q. H. Tran, A relaxation method for two-phase flow models with hydrodynamic closure law, Numer. Math., 99 (2005), pp. 411440.

[3] M. Baudin, F. Coquel, And Q. H. Tran, A semi-implicit relaxation scheme for modeling two-phase flow in a pipeline, SIAM J. Sci. Comput., 27 (2005), pp. 914-936. 
[4] S. Benzoni-Gavage, Analyse Numérique des Modèles Hydrodynamiques d'Écoulements Diphasiques Instationnaires dans les Réseaux de Production Pérolière, PhD dissertation, École Normale Supérieure de Lyon, 1991.

[5] M. Born And L. Infeld, Foundations of a new field theory, Proc. Roy. Soc., (1934), pp. 425451.

[6] F. Coquel and B. Perthame, Relaxation of energy and approximate Riemann solvers for general pressure laws in fluid dynamics, SIAM J. Numer. Anal., 35 (1998), pp. 2223-2249.

[7] S. Evje And T. FlÅTten, Weakly implicit numerical schemes for a two-fluid model, SIAM J. Sci. Comput., 26 (2005), pp. 1449-1484.

[8] I. Faille And E. Heintzé, A rough finite volume scheme for modeling two phase flow in a pipeline, Computers and Fluids, 28 (1999), pp. 213-241.

[9] E. Godlewski and P. A. Raviart, Hyperbolic systems of conservation laws, Mathématiques et Applications, SMAI, Ellipses, 1991.

[10] A. In, Numerical evaluation of an energy relaxation method for inviscid real fluids, SIAM J. Sci. Comput., 21 (1999), pp. 340-365.

[11] S. Jin AND M. Slemrod, Regularization of the Burnett equations for rapid granular flows via relaxation., Physica D, 150 (2001), pp. 207-218.

[12] S. Jin AND Z. P. XIN, The relaxation schemes for systems of conservation laws in arbitrary space dimension, Comm. Pure Appl. Math., 48 (1995), pp. 235-276.

[13] B. Larrouturou, How to preserve the mass fraction positivity when computing multicomponent flows, J. Comput. Phys., 95 (1991), pp. 59-84.

[14] R. J. LeVeque, Numerical Methods for Conservation Laws, Lectures in Mathematics, ETH Zürich, Birkhäuser Verlag, Berlin, 1992.

[15] J. M. Masella, I. Faille, and T. GallouËt, On an approximate Godunov scheme, Int. J. Comput. Fluid Dynam., 12 (1999), pp. 133-149.

[16] J. M. Masella, Q. H. Tran, D. Ferré, and C. Pauchon, Transient simulation of two-phase flows in pipes, Int. J. Multiph. Flow, 24 (1998), pp. 739-755.

[17] C. Pauchon, H. Dhulésia, G. Binh-Cirlot, and J. Fabre, TACITE: a transient tool for multiphase pipeline and well simulation, in SPE annual Technical Conference, New Orleans, 1994.

[18] D. SerRe, Systèmes de Lois de Conservation I $\mathscr{G}$ II, Collection Fondations, Diderot Éditeur Arts et Sciences, Paris, 1996.

[19] J. Whitham, Linear and Nonlinear Waves, New-York, 1974.

[20] N. Zuber and J. Findlay, Average volumetric concentration in two-phase flow systems, J. Heat Transfer, C87 (1965), pp. 453-458.

\section{A Generalized Born-Infeld for an arbitrary flux}

This appendix aims at providing an abstract setting that extends the Born-Infeld relaxation to a general scalar conservation law

$$
\partial_{t} u+\partial_{x} f(u)=0, \quad u \in[0,1]
$$

where the flux $f(u)$ is not necessarily of the form $u(1-u) g(u)$. 


\section{A.1 Admissible changes of variables}

Let us consider the homogeneous Born-Infeld system

$$
\begin{aligned}
& \partial_{t} W+Z \partial_{x} W=0 \\
& \partial_{t} Z+W \partial_{x} Z=0
\end{aligned}
$$

for $(W, Z) \in \bar{\Omega}$. A pair $(E, R)$ of smooth functions of $(W, Z) \in \Omega$ is said to be an entropyflux pair for (A.2) if we have the conservation law

$$
\partial_{t} E(W, Z)+\partial_{x} R(W, Z)=0
$$

Despite the label "entropy," no convexity property is required on $E$.

Lemma A.1. A necessary and sufficient condition for $(E, R)$ to be an entropy-flux pair for the homogeneous Born-Infeld system is

$$
R_{W}=Z E_{W}, \quad R_{Z}=W E_{Z} .
$$

Then, the entropy function E obeys the Goursat equation

$$
E_{W}-E_{Z}+(Z-W) E_{W Z}=0
$$

the general solution of which is

$$
E(W, Z)=\frac{A(W)+B(Z)}{Z-W}, \quad R(W, Z)=\frac{Z A(W)+W B(Z)}{Z-W},
$$

where $A$ and $B$ are two arbitrary smooth functions.

Proof See [18].

To quote a few examples, the pair $(U, F)$ introduced in (2.7) is an entropy-flux pair with $(A, B)=(0, Z)$. The pair $(I, K)=(W+Z, W Z)$, which shortly appeared in the proof of Corollary 2.1, is also an entropy-flux pair with $(A, B)=\left(-W^{2}, Z^{2}\right)$.

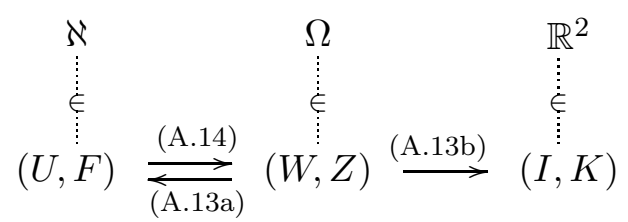

From now on, we consider two general entropy-flux pairs, also denoted by $(U, F)$ and $(I, K)$, as depicted in diagram (A.7). Of course, the first pair $(U, F)$ is meant to be the relaxation counterparts of the equilibrium values $(u, f(u))$. We require that the $F$ can be extended by continuity to all $(W, Z) \in \bar{\Omega}$. We also require that the mapping $(W, Z) \mapsto(U, F)$ be well-defined and invertible for $(W, Z) \in \Omega$, which allows us to consider its inverse $(U, F) \mapsto(W, Z)$, defined over some domain $\aleph \subset[0,1] \times \mathbb{R}$ that we do not seek to clarify further, insofar as our calculations are essentially formal.

Definition A.1. The change of variables $(W, Z) \leftrightarrows(U, F)$ is said to be admissible if

1. Each of the pairs $(U, W)$ and $(U, Z)$ can replace $(U, F)$ as independent variables. In other words,

$$
W_{F}(U, F) \neq 0, \quad Z_{F}(U, F) \neq 0
$$

for all $(U, F)$ under consideration. 
2. The equilibrium speeds, defined as

$$
w(u)=W(u, f(u)), \quad z(u)=Z(u, f(u)),
$$

remain bounded for $u \in[0,1]$.

The second pair $(I, K)$ is intended to play the role of $\left((1-2 U) G,-U(1-U) G^{2}\right)$ in (2.22) of Corollary 2.1. To be more accurate, let us chain the mappings to obtain

$$
\mathscr{I}(U, F)=I(W(U, F), Z(U, F)), \quad \mathscr{K}(U, F)=K(W(U, F), Z(U, F)) .
$$

Definition A.2. The change of variables $(W, Z) \rightarrow(I, K)$ is said to be admissible if

1. The pair $(U, \mathscr{I})$ can replace $(U, F)$ as independent variables. In other words,

$$
\mathscr{I}_{F}(U, F) \neq 0
$$

for all $(U, F)$ under consideration.

2. The equilibrium values for the entropy-flux $(I, K)$, defined as

$$
\mathfrak{I}(u)=\mathscr{I}(u, f(u)), \quad \mathfrak{K}(u)=\mathscr{K}(u, f(u))
$$

remain bounded for $u \in[0,1]$.

For later use, let us specify the compatibility conditions (A.4) as

$$
\begin{array}{ll}
F_{W}=Z U_{W}, & F_{Z}=W U_{Z} \\
K_{W}=Z I_{W}, & K_{Z}=W I_{Z} .
\end{array}
$$

Moreover, as a consequence of (A.13a), we end up with

$$
W_{U}=-W W_{F}, \quad Z_{U}=-Z Z_{F}
$$

by differentiating the inverse mapping $(U, F) \mapsto(W, Z)$.

\section{A.2 Relaxation system}

Our departure point is now the basic variables $(U, F)$, from which we define $(W, Z)$ and $(\mathscr{I}, \mathscr{K})$, in accordance with diagram (A.7) and so as to be endowed with admissible changes of variables in the sense of Definitions A.1 and A.2.

Definition A.3. For a given choice of admissible changes of variables, the generalized Born-Infeld relaxation of equation (A.1) is the system

$$
\begin{aligned}
\partial_{t} W+Z \partial_{x} W & =\lambda W_{F}[f(U(W, Z))-F] \\
\partial_{t} Z+W \partial_{x} Z & =\lambda Z_{F}[f(U(W, Z))-F],
\end{aligned}
$$

for $(W, Z) \in \Omega$, where $\lambda>0$ is the relaxation coefficient. 
In (A.15), the quantities $\left(W_{F}, Z_{F}\right)$ have to be understood as functions of the velocitylike variables $(W, Z)$ via

$$
W_{F}=W_{F}(U(W, Z), F(W, Z)), \quad Z_{F}=Z_{F}(U(W, Z), F(W, Z)) .
$$

Note that the right-hand sides of (A.15) are not written in the same form as those of (2.17). However, they do have the same values when $f=u(1-u) g$ and for the change of variables (2.7)-(2.8).

As in Proposition 2.2, the characteristic fields of the Born-Infeld system (A.15) are linearly degenerate, which enables us to extend results valid for smooth solutions to weak solutions.

Theorem A.1. The generalized Born-Infeld relaxation system (A.15) is equivalent to the conservative form

$$
\begin{aligned}
\partial_{t} U+\partial_{x} F & =0 \\
\partial_{t} \mathscr{I}(U, F)+\partial_{x} \mathscr{K}(U, F) & =\lambda \mathscr{I}_{F}(U, F)[f(U)-F] .
\end{aligned}
$$

Proof To prove (A.17a), multiply (A.15a) by $U_{W},(\mathrm{~A} .15 \mathrm{~b})$ by $U_{Z}$, add them together and make use of (A.13a). The right-hand side vanishes because $U_{W} W_{F}+U_{Z} Z_{F}=U_{F}=0$. Proceed similarly for (A.17b) and invoke the chain rule $I_{W} W_{F}+I_{Z} Z_{F}=\mathscr{I}_{F}$.

\section{A.3 Chapman-Enskog analysis and Whitham condition}

The good news is that the equivalent equation given in Theorem 2.2 is still valid, although its proof now involves more tortuous calculations.

Theorem A.2. At the first order approximation in $\lambda^{-1}$, the solution $u$ to relaxation system (A.17) satisfies the equivalent equation

$$
\partial_{t} u+\partial_{x} f(u)=\lambda^{-1} \partial_{x}\left\{-\left[f^{\prime}(u)-w(u)\right]\left[f^{\prime}(u)-z(u)\right] \partial_{x} u\right\} .
$$

Proof With $u \equiv U$, we insert the standard Chapman-Enskog expansion

$$
F=f(u)+\lambda^{-1} f_{1}+O\left(\lambda^{-2}\right)
$$

into (A.17b). Keeping the leading terms only, while remembering that all functions of $(U, F)$ must be evaluated at $(u, f(u))$, yields

$$
\begin{aligned}
-\mathscr{I}_{F}(u, f(u)) f_{1} & =\partial_{t} \mathscr{I}(u, f(u))+\partial_{x} \mathscr{K}(u, f(u)) \\
& =\mathscr{I}^{\prime}(u, f(u)) \partial_{t} u+\mathscr{K}^{\prime}(u, f(u)) \partial_{x} u \\
& =\left[\mathscr{K}^{\prime}(u, f(u))-f^{\prime}(u) \mathscr{I}^{\prime}(u, f(u))\right] \partial_{x} u
\end{aligned}
$$

where $(.)^{\prime}$ designates the total derivative with respect to $u$, namely,

$$
(.)^{\prime}=\frac{d}{d u}(.)=\partial_{U}(.)+f^{\prime}(u) \partial_{F}(.) .
$$

Inserting (A.21) into (A.20c), and with the help of (A.13b), we obtain

$$
\begin{aligned}
\mathscr{K}^{\prime}-f^{\prime}(u) \mathscr{I}^{\prime} & =\left[K_{W} W^{\prime}+K_{Z} Z^{\prime}\right]-f^{\prime}(u)\left[I_{W} W^{\prime}+I_{Z} Z^{\prime}\right] \\
& =\left[Z I_{W} W^{\prime}+W I_{Z}^{\prime}\right]-f^{\prime}(u)\left[I_{W} W^{\prime}+I_{Z} Z^{\prime}\right] \\
& =\left(z(u)-f^{\prime}(u)\right) W^{\prime} I_{W}+\left(w(u)-f^{\prime}(u)\right) Z^{\prime} I_{Z} .
\end{aligned}
$$


Applying (A.21) again to transform $\left(W^{\prime}, Z^{\prime}\right)$ and invoking (A.14), we end up with

$$
-\mathscr{I}_{F}(u, f(u)) f_{1}=-\mathscr{I}_{F}\left(f^{\prime}(u)-w(u)\right)\left(f^{\prime}(u)-z(u)\right) \partial_{x} u .
$$

After simplification by $\mathscr{I}_{F} \neq 0$, plug $f_{1}$ into (A.17a) and deduce the desired result.

Theorem A.3. A sufficient condition for the relaxation system (A.17) to be a dissipative approximation of the original equation (A.1) is that the Whitham subcharacteristic condition

$$
f^{\prime}(u) \in\lfloor w(u), z(u)\rceil
$$

holds for all $u$ in the range of the problem at hand.

Proof Identical to that of Theorem 2.3.

\section{A.4 Numerical flux and monotonicity}

Like the Born-Infeld relaxation of $\S 2$, the Riemann problem associated with (A.15) can be solved for the initial left and right data

$$
\mathbf{v}_{L}=\left(w\left(u_{L}\right), z\left(u_{L}\right)\right), \quad \mathbf{v}_{R}=\left(w\left(u_{R}\right), z\left(u_{R}\right)\right) .
$$

The intermediate states obey a maximum principle componentwise, as in Theorem 2.4. As in Theorem 2.5, the flux $F^{*}$ at $x=0$ always makes sense, and satisfies a sign property. This allows us to consider the numerical flux

$$
H^{\mathrm{BI}}\left(u_{L}, u_{R}\right)=\mathscr{F}\left(\mathbf{v}_{L}, \mathbf{v}_{R}\right)=F^{*}
$$

and to ask question about the equivalence between the monotonicity of $H^{\mathrm{BI}}$ and the Whitham condition (A.24), as was the case in Theorem 2.6.

Theorem A.4. Assume that each component of the pair $(w(u), z(u))$ keeps a constant sign over $u \in] 0,1\left[\right.$. Then a necessary condition for the Born-Infeld numerical flux $H^{\mathrm{BI}}\left(u_{L}, u_{R}\right)$ to be monotonous for all $\left.\left(u_{L}, u_{R}\right) \in\right] 0,1\left[^{2}\right.$ is that the Whitham condition (A.24) is satisfied for all $u \in] 0,1[$.

This condition is also sufficient if each component of the pair $\left(U_{W}(W, Z), U_{Z}(W, Z)\right)$ keeps a constant sign for all $(W, Z)$ at issue.

Proof Without loss of generality, suppose we are in Case $1 \mathrm{a}$ of Fig. 2, that is, $w<0$ and $z>0$. Then the intermediate state is $\mathbf{v}^{*}=\widetilde{\mathbf{v}}=\left(w\left(u_{L}\right), z\left(u_{R}\right)\right)$, and

$$
H^{\mathrm{BI}}\left(u_{L}, u_{R}\right)=F\left(w\left(u_{L}\right), z\left(u_{R}\right)\right) .
$$

By differentiation,

$$
H_{u_{L}}^{\mathrm{BI}}=F_{W}\left(w\left(u_{L}\right), z\left(u_{R}\right)\right) w^{\prime}\left(u_{L}\right)=z\left(u_{L}\right) U_{W}\left(w\left(u_{L}\right), z\left(u_{R}\right)\right) w^{\prime}\left(u_{L}\right)
$$

where we have used (A.13a). Let

$$
\mathfrak{b}\left(u_{L}, u_{R}\right)=U_{W}\left(w\left(u_{L}\right), z\left(u_{R}\right)\right) w^{\prime}\left(u_{L}\right) .
$$

Then,

$$
H_{u_{L}}^{\mathrm{BI}}=\mathfrak{b}\left(u_{L}, u_{R}\right) z\left(u_{L}\right)
$$


Similarly, we have

$$
H_{u_{R}}^{\mathrm{BI}}=\mathfrak{a}\left(u_{L}, u_{R}\right) w\left(u_{R}\right)
$$

with

$$
\mathfrak{a}\left(u_{L}, u_{R}\right)=U_{Z}\left(w\left(u_{L}\right), z\left(u_{R}\right)\right) z^{\prime}\left(u_{R}\right) .
$$

Therefore, the monotonicity conditions $H_{u_{L}}^{\mathrm{BI}} \geq 0$ and $H_{u_{R}}^{\mathrm{BI}} \leq 0$ are tantamount to

$$
\mathfrak{a}\left(u_{L}, u_{R}\right) \geq 0, \quad \mathfrak{b}\left(u_{L}, u_{R}\right) \geq 0 .
$$

Taking the derivatives of

$$
u=U(w(u), z(u)), \quad f(u)=F(w(u), z(u))
$$

with respect to $u$, we obtain

$$
\begin{array}{rlrl}
1 & = & U_{W}(w(u), z(u)) w^{\prime}(u)+ & U_{Z}(w(u), z(u)) z^{\prime}(u) \\
f^{\prime}(u) & = & F_{W}(w(u), z(u)) w^{\prime}(u)+ & F_{Z}(w(u), z(u)) z^{\prime}(u) \\
& =z(u) U_{W}(w(u), z(u)) w^{\prime}(u)+w(u) U_{Z}(w(u), z(u)) z^{\prime}(u),
\end{array}
$$

again because of (A.13a). With the notations (A.29), (A.32), this system can be cast under the form

$$
\begin{aligned}
1 & =\quad \mathfrak{a}(u, u)+\quad \mathfrak{b}(u, u) \\
f^{\prime}(u) & =w(u) \mathfrak{a}(u, u)+z(u) \mathfrak{b}(u, u) .
\end{aligned}
$$

The Whitham condition (A.24) says that $f^{\prime}(u)$ must be a convex combination of $w(u)$ and $z(u)$. In view of (A.36), this is equivalent to

$$
\mathfrak{a}(u, u) \geq 0, \quad \mathfrak{b}(u, u) \geq 0 .
$$

It is plain that

$$
\text { (A.33) for all } \left.\left(u_{L}, u_{R}\right) \in\right] 0,1\left[^{2} \Longrightarrow(\text { A.37) for all } u \in] 0,1[\text {. }\right.
$$

Hence, monotonicity of the numerical flux implies the Whitham condition. Furthermore, from the definitions (A.29), (A.32), we clearly see that if $\left(U_{W}, U_{Z}\right)$ keeps a constant sign componentwise, the monotonicity condition (A.33) splits into two separated and decoupled conditions, one on $z^{\prime}\left(u_{L}\right)$, the other on $w^{\prime}\left(u_{R}\right)$. The Whitham condition (A.37) also splits into two conditions, one on $z^{\prime}(u)$, the other on $w^{\prime}(u)$. In this case, we have the converse

$$
\text { (A.37) for all } u \in] 0,1\left[\Longrightarrow\left(\text { A.33) for all }\left(u_{L}, u_{R}\right) \in\right] 0,1\left[^{2}\right. \text {, }\right.
$$

which completes the proof. 